\title{
SMF-1, SMF-2 and SMF-3 DMT1 Orthologues Regulate and Are Regulated Differentially by Manganese Levels in C. elegans
}

\author{
Catherine $\mathrm{Au}^{1,29}$, Alexandre Benedetto ${ }^{1,3,89}$, Joel Anderson ${ }^{4 \uparrow}$, Arnaud Labrousse ${ }^{5,6,7,9 \uparrow}$, Keith Erikson ${ }^{4}$, \\ Jonathan J. Ewbank ${ }^{5,6,7}$, Michael Aschner ${ }^{1,2,3 *}$
}

1 Department of Pediatrics, Vanderbilt University, Nashville, Tennessee, United States of America, 2 Center for Molecular Toxicology, Vanderbilt University, Nashville, Tennessee, United States of America, 3 Children's Hospital, Vanderbilt University, Nashville, Tennessee, United States of America, 4 Department of Nutrition, The University of North Carolina Greensboro, Greensboro, North Carolina, United States of America, $\mathbf{5}$ Centre d'Immunologie de Marseille-Luminy, Université de la Méditerranée, Marseille, France, 6 U631, INSERM, Marseille, France, 7 UMR6102, CNRS, Marseille, France, 8 London Centre for Nanotechnology, University College London, London, United Kingdom, $\mathbf{9}$ Institut de Pharmacologie et de Biologie Structurale, Université Paul Sabatier Toulouse III, Toulouse, France

\begin{abstract}
Manganese $(\mathrm{Mn})$ is an essential metal that can exert toxic effects at high concentrations, eventually leading to Parkinsonism. A major transporter of $\mathrm{Mn}$ in mammals is the divalent-metal transporter (DMT1). We characterize here DMT1-like proteins in the nematode $C$. elegans, which regulate and are regulated by $\mathrm{Mn}$ and iron (Fe) content. We identified three new DMT1-like genes in C. elegans: smf-1, smf-2 and smf-3. All three can functionally substitute for loss of their yeast orthologues in S. cerevisiae. In the worm, deletion of smf-1 or smf-3 led to an increased Mn tolerance, while loss of smf-2 led to increased Mn sensitivity. smf mRNA levels measured by QRT-PCR were up-regulated upon low $\mathrm{Mn}$ and down-regulated upon high Mn exposures. Translational GFPfusions revealed that SMF-1 and SMF-3 strongly localize to partially overlapping apical regions of the gut epithelium, suggesting a differential role for SMF-1 and SMF-3 in Mn nutritional intake. Conversely, SMF-2 was detected in the marginal pharyngeal epithelium, possibly involved in metal-sensing. Analysis of metal content upon Mn exposure in smf mutants revealed that SMF3 is required for normal $\mathrm{Mn}$ uptake, while $s m f-1$ was dispensable. Higher smf-2 mRNA levels correlated with higher Fe content, supporting a role for SMF-2 in Fe uptake. In smf-1 and smf-3 but not in smf-2 mutants, increased Mn exposure led to decreased Fe levels, suggesting that both metals compete for transport by SMF-2. Finally, SMF-3 was post-translationally and reversibly down-regulated following Mn-exposure. In sum, we unraveled a complex interplay of transcriptional and post-translational regulations of 3 DMT1-like transporters in two adjacent tissues, which regulate metal-content in C. elegans.
\end{abstract}

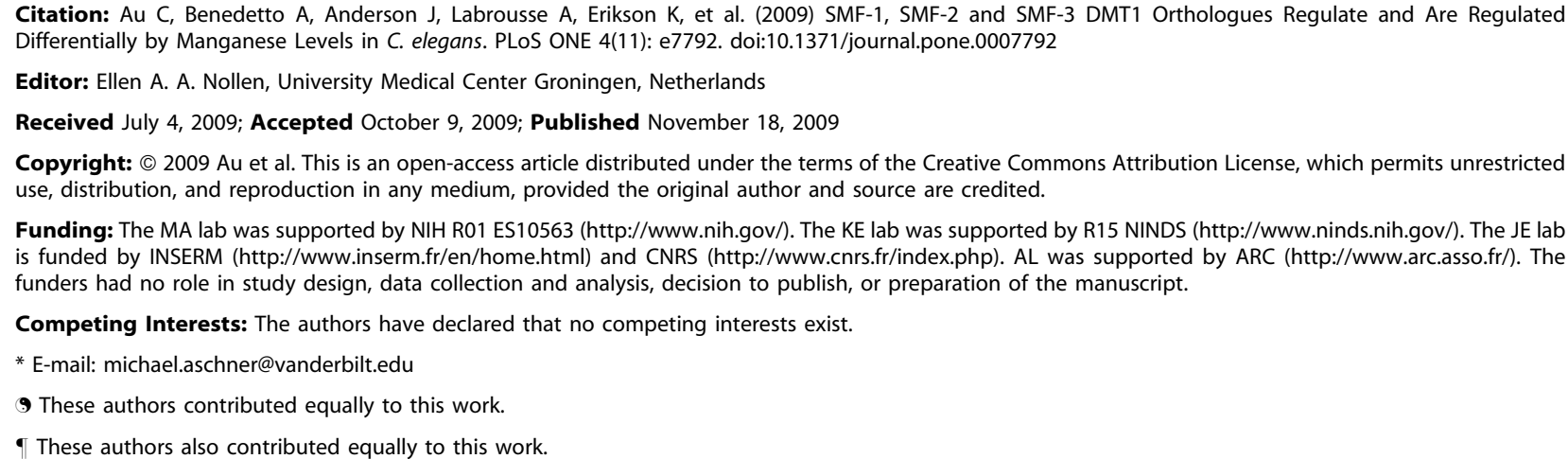

Funding: The MA lab was supported by NIH R01 ES10563 (http://www.nih.gov/). The KE lab was supported by R15 NINDS (http://www.ninds.nih.gov/). The JE lab is funded by INSERM (http://www.inserm.fr/en/home.html) and CNRS (http://www.cnrs.fr/index.php). AL was supported by ARC (http://www.arc.asso.fr/). The funders had no role in study design, data collection and analysis, decision to publish, or preparation of the manuscript.

Competing Interests: The authors have declared that no competing interests exist.

*E-mail: michael.aschner@vanderbilt.edu

9 These authors contributed equally to this work.

- These authors also contributed equally to this work.

\section{Introduction}

Manganese $(\mathrm{Mn})$ is one of the most abundant natural elements in the Earth's crust. It most frequently occurs in the form of oxides, carbonates and silicates [1]. It is one of 7 essential metals for animals, acting as a cofactor for multiple proteins with a wide variety of biological activities, such as transferases, hydrolases, lyases, arginase, glutamine synthetase, superoxide dismutase and integrins [2,3]. Consequently, $\mathrm{Mn}$ is essential for many physiological processes, such as modulation of the immune system, stellate process production in astrocytes, as well as protein, lipid and carbohydrate metabolism $[4,5,6,7,8]$. Mn is also essential for optimal brain and skeletal structure development [9,10]; Mn deficiency has been associated with defective bone formation and increased susceptibility to seizures $[11,12]$. Despite being essential for metabolic functions, $\mathrm{Mn}$ at high concentrations can be toxic, especially to the brain. Though most Mn is obtained through the diet, Mn toxicity from dietary intake is rare, because $\mathrm{Mn}$ balance is tightly regulated by both the enterocytes (intake) and the biliary duct cells (excretion). In contrast, pulmonary uptake and particulate transport via the olfactory bulb $[2,13,14]$ can lead to deposition of $\mathrm{Mn}$ within the striatum and cerebellum, and inflammation of the nasal epithelium [15]. Exposure to excessive Mn levels may cause an extrapyramidal syndrome, referred to as manganism, closely resembling idiopathic Parkinson's disease (IPD), both at the molecular and clinical level $[16,17,18]$. Mn exposure represents a significant public health matter due to the use of $\mathrm{Mn}$ as a catalyser in countless industrial processes, its presence in gasoline additive, fungicides such as Maneb and in permanganate, a drinking water purifier [1,2,19,20,21]. Because $\mathrm{Mn}$ is the only environmental toxicant that has been robustly associated with IPD, studies on the mechanisms that mediate its 
toxicity offer means for better understanding of neurodegenerative diseases, the frequency of which is on the rise [22,23,24].

Due to the delicate relationship between essentiality and toxicity, Mn homeostasis is crucial for all eukaryotes. Previous research has focused on Mn transport mechanisms across the blood-brain barrier (BBB), but the nature and relative contributions of the carrier(s) identified thus far remain controversial. Over the past two decades, active transport [25] and facilitated diffusion [26,27] mechanisms have been described. More recently, Mn transport has been ascribed to high affinity metal transporters of calcium $(\mathrm{Ca})$ and iron $(\mathrm{Fe})$. Amongst these, attention has been directed to the divalent metal transporter (DMT1 [28]), which belongs to the family of natural resistance-associated macrophage protein (NRAMP) $[29,30,31,32]$. To avoid confusion, we will further refer to NRAMP family members as DMT(s) for Divalent Metal Transporter(s).

DMT1, previously known as NRAMP2, was first identified as an orthologue of NRAMP1, a protein involved in host defense against several types of infection [29,30]. Subsequently, it was referred to as divalent cation transporter (DCT1), because of its ability to transport divalent zinc $\left(\mathrm{Zn}^{2+}\right)$, manganese $\left(\mathrm{Mn}^{2+}\right)$, cobalt $\left(\mathrm{Co}^{2+}\right)$, cadmium $\left(\mathrm{Cd}^{2+}\right)$, copper $\left(\mathrm{Cu}^{2+}\right)$, nickel $\left(\mathrm{Ni}^{2+}\right)$, lead $\left(\mathrm{Pb}^{2+}\right)$, and iron $\left(\mathrm{Fe}^{2+}\right)[32,33,34]$. In 1999 it was designated as DMT1 [28]. DMT1 is an integral membrane protein conserved from bacteria to humans, containing 11 to 12 transmembrane domains (TMD) and a "consensus transport sequence" (CTS) involved in divalent metal ion transport $[32,33,34,35]$. Notably, rodent models presenting a spontaneous mutation in DMT1, microcytic (mk) mice and Belgrade $(\mathrm{b} / \mathrm{b})$ rats [36], and several human mutations in DMT1 [37,38,39,40,41,42] suggest an association between microcytic anemia, impairment of $\mathrm{Fe}$ transport and $\mathrm{Mn}$ homeostasis. In vertebrates, DMT1 is ubiquitously expressed, but more abundant expression is observed in the proximal duodenum compared to the kidney or the brain $[28,32,43]$. Within the brain, the basal ganglia express higher levels of DMT1, where Mn preferentially accumulates [44]. At a subcellular level, DMT1 is strongly localized at the apical membrane in enterocytes [45] and sustentacular cells of the olfactory epithelium [14] whereas in macrophages, it is restricted to the phagosomal membrane. The vertebrate DMT1 gene produces four alternatively spliced mRNAs differing by the presence or absence of the exon 0 , and a $3^{\prime}$ sequence in which an iron regulatory element is present or absent (+IRE and -IRE respectively). The $3^{\prime}$ UTR is responsible for the modulation of the +IRE mRNA stability by the intracellular Fe pool [32]. The +IRE vs -IRE mRNAs encode DMT1 isoforms with distinct carboxy- termini $[43,46]$. The +IRE isoforms are found mainly at the apical membrane of epithelial cells $[45,47]$ and in late endosomes and lysosomes within HEp-2 cells $[40,48]$. The -IRE isoforms are found predominantly in early and recycling endosomes [49,50,51]. Studies in yeast have identified three DMT1 orthologues: Smflp, Smf2p and Smf3p, encoded by $S M F 1, S M F 2$ and $S M F 3$ respectively. In contrast to vertebrate DMT1, no typical IRE is found in any of the $S M F$ genes, and the poor sequence conservation of the C-termini is not predictive of a correspondence between the vertebrate -IRE/ + IRE isoforms and Smflp/2p/3p. However, Smflp was identified as a non-specific metal ion transporter for $\mathrm{Mn}^{2+}, \mathrm{Zn}^{2+}, \mathrm{Cu}^{2+}, \mathrm{Fe}^{2+}$ and $\mathrm{Cd}^{2+}[52,53]$ and Smf2p was also found to be involved in $\mathrm{Mn}$ transport [54]. Analogous to their vertebrate orthologues, the yeast DMTs are found in various intracellular compartments: Smflp at the cell surface, Smf2p in vesicles and Smf3p exclusively at the vacuolar membrane $[53,55,56]$. In addition, they differentially contribute to $\mathrm{Mn}$ homeostasis, since Smfpl and Smf3p are dispensable in Mn-replete conditions [53], while Smf2p is essential for ensuring proper Mn uptake [57,58]. Additionally, the SMF3 promoter is found to contain a target sequence for the Fe-sensing transcription factor Aftlp, which is responsible for its transcriptional regulation by $\mathrm{Fe}$, whereas $\mathrm{Smflp}$ and $\mathrm{Smf} 2 \mathrm{p}$ levels are unaffected by Fe concentration $[55,56]$.

Currently, both Mn transport and DMT protein function(s) are poorly understood. This is partly due to the existence of several DMT proteins differentially regulated at the transcriptional and post-translational levels, in distinct tissues (intestine, liver, kidney, brain) and under various conditions ( $\mathrm{Mn}$ or Fe levels, infection). Moreover, findings in a unicellular organism like the baker's yeast are difficult to translate to metazoans and especially to mammals. Given the current lack of a genetically amenable animal model, we used the C. elegans system to address these issues. We identified and cloned three functional C. elegans DMT1 orthologues SMF-1, SMF-2 and SMF-3, with distinct roles in $\mathrm{Mn}$ transport regulation. Our results support an evolutionary conserved function for DMT1 isoforms in the regulation of $\mathrm{Mn}$ uptake, and emphasize the differential contribution and regulation of DMT1 isoforms expressed in different tissues and exhibiting different subcellular localizations. Furthermore, our study identifies SMF-3 as the main Mn uptake transporter in the worm, whereas SMF-1 has a minor role in this process and SMF-2 is involved in metal content regulation.

\section{Methods}

Multiple alignment generation and analysis. - Protein sequences were uploaded from the NCBI website (http://www.ncbi.nlm.nih.gov/ sites/entrez). Multiple alignments and phylogenetic trees were generated using ClustalX1.81 [59] running ClustalW [60].

Yeast handling, transformation and EGTA sensitivity assessment. - Yeast strains, maintenance, transformation and culture for the EGTA sensitivity assay were performed as described [58]. Plasmids pVTCesmf-1, pVT-Cesmf-2 and pVT-Cesmf-3, for yeast expression of C. elegans genes smf-1, smf-2 and smf-3 respectively, were generated as follows. smf-1 (from yk452d4) and smf-3 (from yk397h3) cDNA were kindly provided by Yuji Kohara, while $s m f-2$ cDNA was obtained by reverse-transcription-PCR. smf-1 was amplified using primers $5^{\prime}$ CAG CGG ATC CGC TTG ATA TCG TGG ATT GTC-3' and 5'-GAC CGG TAC CGG AAA GTA TAC ATC GTT CAC-3'. smf-3 was amplified using primers $5^{\prime}$-CGCGGATCGAATGGAGGTGAAATGAAAT- $3^{\prime}$ and 5'-GCGCGGTACGGCATATCGCATAGACAGTTC-3'. smf-1' smf-2, and smf-3 fragments were then cloned into pVT101-U using HindIII and PstI.

C. elegans strains and handling of the worms. - C. elegans strains were handled and maintained at $20^{\circ} \mathrm{C}$ as previously described [61]. The following strains were used: $\mathrm{N} 2$ (wildtype); IG6, smf-1(eh5) $X$; VC171, smf-2(gk133) X; RB1074, smf-3(ok1035) IV; MT455, lon2(e678) unc-18(e81) X; MT628, dpy-9(e12) unc-17(e245) IV. All strains were provided by the Caenorhabditis Genetic Center (CGC, Minnesota). The IG6, VG171 and RB1074 strains were outcrossed 4 additional times to generate MAB23, MAB21 and MAB37, respectively.

Gene cloning and plasmid constructions. - Restriction enzymes were ordered from New England Biolabs. PCR amplifications used the LA Taq ${ }^{\text {TM }}$ Polymerase (Takara), and were performed on an Eppendorf2 PCR-machine. Translational C-terminal GFP-fusions for SMF-1, SMF-2 and SMF-3 localization were generated as follow. The smf-1 ORF and preceding intergenic region $(4.4 \mathrm{~kb})$ was amplified using primers: 5'- TAT TAC CTG CAG GAG CTA GGT TCA TGT TCA GCG CGA AGC TGG-3', and 5'TAT TAG GAT CAA ATT GAT ATC CTG CAT TGT GAT GGA CTGC-3'. This fragment was cloned into the pPD95.75 vector (Fire kit) between the SbfI and BamHI restriction sites, in frame with the GFP (plasmid pMA0015). The smf-2 ORF with its 
preceding intergenic sequence $(3.5 \mathrm{~kb})$ was amplified using primers: 5'-TAT TGT GGA GTC ATA GGA AAA GGA TGG TCG GTG-3' and 5'-TAT TGG TAC CAG AAA GTA TAC ATC GTT CAC AAC-3'. The smf-3 ORF and its preceding intergenic region $(8.4 \mathrm{~kb})$ were amplified using primers: $5^{\prime}$-TAT TCT GCA GAC TTC ATT GGG GAT GTG CTT TGG-3' and 5'-GGT ACG CAA TAT CGC ATA GAG AGT TGG TCG-3'. The smf-2 and smf-3 amplification products were then cloned into pPD95.75 between the PstI and KpnI restriction sites, in frame with the GFP (plasmid pMA0010 and pMA0004 respectively). Transcriptional constructs for $s m f-1, s m f-2$ and $s m f-3$ were obtained by cloning the preceding intergenic region, respectively up to the $2^{\text {nd }}, 6^{\text {th }}$ and $7^{\text {th }}$ exon to include highly conserved intronic sequences. The following primer sets were used: 5'-CGG AAG CTT CGA GGA GCT CGG ATT G-3' and 5'-CGC CTG CAG CCT TGT GCG CAA GAC TGA AGG-3' for $s m f-1,5^{\prime}$-TAT TGG ATG CTC ATA CGA AAA CGA TGG TCG GTG-3' and 5'-TAT TCT GCA GTA GTC CAA ACT GAC ATC CGA GG-3' for smf-2, 5' -TAT TGT GCA GAC TTC ATT GGG GAT GTG CTT TGG-3' and 5'-TAT TGT CGA CGG CTC TGG AAT ATA ATT AGG ATT GC-3' for smf-3. The smf-1 product $(1.7 \mathrm{~kb})$ was cloned between the HindIII and PstI restriction sites in pPD95.75 (promSmflGFP1). The smf-2 (2.6 kb) fragment was cloned into pPD95.69 (Fire kit) between the SphI and PstI restriction sites (pMA0009). The smf-3 (5.5 kb) fragment was cloned into pPD95.69 between the restriction sites PstI and SalI (pMA0003).

C. elegans transgenesis. - DNA was injected into the syncytial gonads of $\mathrm{N} 2$ hermaphrodites using injection mixes containing $25 \mathrm{ng} / \mu \mathrm{l}$ construct and $175 \mathrm{ng} / \mu \mathrm{l} \mathrm{pRF} 4$ as a transformation marker [62]. A minimum of three independent transgenic strains per construct showing the same expression pattern were observed. One strain for each construct was selected for this study: MAB111, mjaEx074[SMF-1::GFP; rol-6(su1006)]; MAB120, mjaEx083[SMF2::GFP; rol-6(su1006)]; MAB105, mjaEx068[SMF-3::GFP; rol6(su1006)].

Acute manganese chloride treatments. - Manganese chloride $\left(\mathrm{MnCl}_{2}\right)$ solutions were prepared in $85 \mathrm{mM} \mathrm{NaCl}$. For each tested strain, 5000 synchronized L1 worms per tube were exposed to 0 to $4 \mathrm{M}$ of $\mathrm{MnCl}_{2}$ in siliconized tubes for 30 minutes. Each condition was performed in tetraplicates. Worms were then pelleted by centrifugation at $7000 \mathrm{rpm}$ for 3 minutes and washed 5 times in $85 \mathrm{mM} \mathrm{NaCl}$. Worms from each of the siliconized tubes were then transferred into individual $100 \mathrm{~mm}$ OP50-1 coated NGM plates, which were then blinded. At $24 \mathrm{~h}$ post-treatment, for each plate, live worms were scored in 4 random $1 \mathrm{~cm}^{2}$ grids to estimate the total number of surviving worms (up to 800 counts per plate). Scores were normalized to percent control ( $\begin{array}{llll}0 & \mathrm{mM} & \mathrm{MnCl}_{2}\end{array}$ exposure).

$\mathrm{Mn}$ and $\mathrm{Fe}$ content measurement by atomic absorption spectrophotometry $(A A S)$. - Triplicates of $7000 \mathrm{~L} 1$ worms per condition were treated with $\mathrm{MnCl}_{2}$ as previously described. The samples were washed 8 times in $85 \mathrm{mM} \mathrm{NaCl}$. The samples were dehydrated in a vacuum oven at $65^{\circ} \mathrm{C}$ for 2 hours, and further digested in $200 \mu \mathrm{l}$ ultrapure nitric acid for 24 hours in a sand-bath $\left(60^{\circ} \mathrm{C}\right)$. A $20 \mu$ l aliquot of the digested sample was brought to $1 \mathrm{ml}$ total volume with $2 \%$ nitric acid and analyzed for $\mathrm{Mn}$ and $\mathrm{Fe}$ content using graphite furnace atomic absorption spectroscopy (AAS) (Varian AA240, Varian, Inc USA). Bovine liver digested in ultrapure nitric acid was used as an internal standard for analysis (NBS Standard Reference Material, USDC, Washington, DC, diluted at $5 \mu \mathrm{g}$ $\mathrm{Mn} / \mathrm{L}$ and $92 \mu \mathrm{g} \mathrm{Fe} / \mathrm{L})$.

Epifluorescence, DIC and confocal microscopy. - For each slide, at least 30 worms were mounted on $4 \%$ agarose pads in M9, and anaesthetized with $0.2 \%$ tricaine $/ 0.02 \%$ tetramisole in M9. Fluorescence observations and DIC imaging were performed with an epifluorescence microscope (Nikon Eclipse 80i, Nikon) equipped with a Lambda LS Xenon lamp (Sutter Instrument Company) and Nikon Plan Fluor 20x dry and Nikon Plan Apo 60x 1.3 oil objectives. The microscope was coupled to a black-andwhite camera (DS-QilMc; Nikon) operated by the Nikon Elements AR3.0 software (NES AR3.0) for image acquisitions. Confocal images acquired for illustration or GFP intensity measurement purposes were captured through Plan-Neofluar $40 \times$, Plan-Apochromat $63 x$, or Plan-Neofluar $100 \times$ oil objectives with a $1.3,1.4$ and 1.3 apertures, respectively, on a LSM510 confocal microscope (Carl Zeiss MicroImaging, Inc.), scanning every $200 \mathrm{~nm}$ for XZ sections. Images were processed with the Zeiss LSM Image Browser 4.0.0.157 software and edited using Photoshop 7.0 (Adobe). Microscopes were in air-conditioned rooms $\left(20-22^{\circ} \mathrm{C}\right)$.

Phenotypic characterization of Mn treated worms.- Following $\mathrm{Mn}$ treatment up to $24 \mathrm{~h}$ post-treatment, worms were observed under stereomicroscope (Zeiss), and mounted for microscopy phenotypic analysis using our Nikon platform aforementioned. After $24 \mathrm{~h}$ at $20^{\circ} \mathrm{C}$, at least 30 random control worms and 30 treated worms exposed to $35 \mathrm{mM} \mathrm{MnCl}$, were imaged at 20x. We assessed their developmental stage (L1 or L2) and their size using NES AR3.0.

SMF-3::GFP fluorescence measurements. - SMF-3::GFP transgenic worms (MAB105) were acutely treated as described previously, transferred on OP50-1 seeded NGM plates and imaged at $1 \mathrm{~h}, 5 \mathrm{~h}$ and $30 \mathrm{~h}$ post-treatment. Fluorescence measurements of SMF3::GFP signal were performed on complete confocal Z-stack projections of $C$. elegans gut. 5 to 12 animals of the same age were imaged for each condition. Treated and untreated animals were mounted on the same slide, and imaged with the same magnification, gain, offset, pinhole and laser power settings. Due to the very small signal/noise ratio for treated animals at $5 \mathrm{~h}$, those settings allowed up to $30 \%$ signal saturation for some of the $5 \mathrm{~h}$, $30 \mathrm{~h}$ control worms and $30 \mathrm{~h}$ recovering worms, so that fluorescence measurements for these conditions are underestimates. Mean signal intensity of the maximal projection of the apical membrane of the intestine (from the pharyngeal-intestinal valve to the rectum) was measured using the freeware ImageJ.

Statistics. - Dose-response lethality curves and histograms for Mn content measurements were generated using GraphPad Prism (GraphPad Software Inc.). We used a sigmoidal dose-response model with a top constraint at $100 \%$ to draw the curves and determine the $\mathrm{LD}_{50}$ values. Statistical analysis of significance was carried out by one-way ANOVA for the dose-response curves, and two-way ANOVA for Mn content measurements followed by posthoc Bonferroni test when the overall $\mathrm{p}$ value was less than 0.05 . For $\mathrm{Fe}$ content, Fisher test revealing differences in variances between the groups, two-tailed unpaired T-test with Welch's correction were used to assess differences in Fe content. In all figures, error bars represent SEM, * p<0.05, ** $\mathrm{p}<0.01$, $* * * \mathrm{p}<0.001$

RNA isolation, cDNA preparation and Real-time PCR. - 10000 N2, MAB21, MAB23 and MAB37 synchronized L1 worms per tube were acutely treated with $\mathrm{MnCl}_{2}$ concentrations of $0,0.1,10$ and $100 \mathrm{mM}$, washed in $\mathrm{NaCl} 85 \mathrm{mM}$, pelleted and frozen in liquid nitrogen. They were thawed on ice, frozen again in $\mathrm{N}_{2 l i q}$ and resuspended in $100 \mu \mathrm{L} \mathrm{H} \mathrm{H}_{2} \mathrm{O}$ and $350 \mu \mathrm{L}$ Trizol (Invitrogen Life Technologies) for RNA isolation. RNA extracts were purified using Qiagene RNeasy mini-kit (Qiagen). Messenger RNAs were reversetranscribed with oligo-dT primers using the Superscript III RNAse H Reverse transcription kit (Invitrogen). cDNAs were stored at $-20^{\circ} \mathrm{C}$. Quantitative Real-time-PCR was carried on a ABISystems HT7900, 
using Brilliant SYBR Green I kit (Stratagene/Agilent Technologies Inc.), and HPLC purified primers (Operon) at a final concentration of $120 \mathrm{nM}$. For amplification of $s m f-1 \mathrm{cDNA}$, two sets of $22 \mathrm{~b}$ primers were designed over the last intron $5^{\prime}$-GCT CGG ATG ACG TTT GCA TAC G-3' / 5' -ATC CTC GGA TGG AAA CGG TGT C-3', and between exons 6 and $85^{\prime}$-TTT CGC ACA TGG ACT TTA CGA G-3' /5' - GCA ATA GCT CGA AAC TGG CAT C-3'. For smf-2, primers over intron 6 5'-TAT TCG CAG CAG GAC AAT CAT C-3' /5'-TTG TGG ATA ATC GGG TTA CTG G-3', and over intron 10 5'-GTT GCT TGC GAA CTT ATG AAC G-3' / 5' ACA AAG GTT TCT GTG ATC CAC G-3', were used. Smf-3 cDNA was amplified using primer sets 5'-TCC AGT GCT GAC ATT TGT ATC G-3' /5'-CAA GGA AAT CAC AAT GGA GAC C-3' over intron 10 and 5'-GGT CTC CAT TGT GATT TCG TTG-3' $/ 5^{\prime}$-CGA AAT CGT GGT AGA TGG GCT CG-3' over intron 11. $c d c-42$ (primers: 5'-CTG CTG GAC AGG AAG ATT ACG-3'/5'-CTC GGA CAT TCT CGA ATG AAG-3') and Y45F10D4F.3 (primers: 5'-GTC GCT TCA AAT CAG TTC AGC3'/5'-GTT CTT GTC AAG TGA TCG GAC A-3'), were used as controls to normalize smf expression levels as they were proven to have stable expression levels independent of age or stress conditions [63]. The amplification setup included $10^{\prime}$ denaturation at $95^{\circ} \mathrm{C}, 20$ cycles at $95^{\circ} \mathrm{C}$ for $30^{\prime \prime} / 58^{\circ} \mathrm{C}$ for $1^{\prime} / 70^{\circ} \mathrm{C}$ for $1^{\prime}, 20$ to 25 cycles at $95^{\circ} \mathrm{C}$ for $30^{\prime \prime} / 56^{\circ} \mathrm{C}$ for $45^{\prime \prime} / 72^{\circ} \mathrm{C}$ for $45^{\prime \prime}$, and a dissociation curve to ascertain that the signal did not result from primer annealing.

\section{Results}

The $\mathrm{C}$. elegans genome encodes 3 DMT1 orthologues: $S M F-1, S M F-2$, and $S M F-3$. A search on the C. elegans genome using the human DMT1 protein sequence revealed three loci encoding DMT1 orthologues: Y69A2AR.4/smf-3 on chromosome IV, and K11G12.3/smf-2 and K11G12.4/smf-1 on X chromosome. All three genes encode proteins with a high degree of conservation (Figure 1). Phylogenetic analysis placed them closer to their insects and vertebrates orthologues than plant and yeast divalent-metal transporters (Fig. 1A). A multiple alignment of the C. elegans SMF1, SMF-2, SMF-3 with their insect, vertebrates and yeast orthologues confirmed this analysis. All three C. elegans SMF exhibit a consensus transport sequence (CTS) quasi-identical to their vertebrate orthologues and 12 transmembrane domains (TMD), unlike the yeast SMF, in which the $11^{\text {th }}$ TMD is absent and the CTS is conserved at $70 \%$ (Fig. 1B).

$\mathrm{SMF}-1, \mathrm{SMF}-2$ and $\mathrm{SMF}-3$ rescue the sensitivity to EGTA of the yeast $\triangle S M F 1+2$ mutant. In the yeast $S$. cerevisiae, Smflp and $\mathrm{Smf} 2 \mathrm{p}$ are divalent-metal transporters that can function in Mn uptake. Double mutants for $\triangle S M F 1+2$ are hypersensitive to the divalentmetal ion scavenger ethylene glycol tetra-acetic acid (EGTA). To assay the molecular function of $C$. elegans SMF-1, -2 and -3 proteins as divalent-metal transporters, we transvected the cDNA encoding each of the C. elegans proteins into the $\triangle S M F 1+2$ yeast mutant, and tested these transgenic strains for hypersensitivity to EGTA. All three $C$. elegans SMF were able to rescue the hypersensitivity to EGTA of the $\triangle S M F 1+2$ mutants (Fig. 2). Moreover, the efficacy of the rescue was at least equivalent to the rescue conferred by transvection of the mouse NRAMP2 cDNA construct [58]. Noticeably, the most potent effect was obtained with smf-1 cDNA, which led to a hyper-resistant phenotype to EGTA. These results indicate that C.elegans SMF-1, SMF-2 and SMF-3 are functionally similar to their yeast and mammal orthologues and are likely involved in divalent-metal ion transport.

Mn exposure can induce lethal osmoregulation defects and a developmental delay in wild type worms. Acute exposure (30 minutes) of wild-type $C$. elegans to $\mathrm{Mn}$ was lethal at concentrations greater than $10 \mathrm{mM}$.
Shortly after treatment and dependent upon the exposure level, a fraction of the worms displayed vacuoles in the main epithelia: epidermis, excretory cell and gut, tissues whose integrity is essential for worm survival (Fig. 3). Loss of the excretory cell was the likely cause of death, since dying worms exhibited the characteristic rodlike phenotype (Fig. $3 F \& G$ ) inherent to excretory-cell defective mutants [64] and excretory-cell ablated worms [65]. Surviving worms at $24 \mathrm{~h}$ were about $70 \%$ shorter $($ Fig. $3 \mathrm{H})$ and displayed an obvious developmental delay when compared with control animals. Twenty-four hours post-treatment $83 \%$ of survivors exposed to a $35 \mathrm{mM} 30 \mathrm{~min} \mathrm{Mn}$ treatment were still at the Ll stage, compared to $13 \%$ in the control group (Fig. 3I). The lethal concentration $50 \%\left(\mathrm{LD}_{50}\right.$ at which half the worms are dead 24 hours after treatment) was $47 \mathrm{mM}$ for the wild-type Bristol N2 strain (Fig. 4).

The deletion-mutants smf- 1 (eh5) and smf-3(ok1035) are hyper-resistant to Mn exposure. In rodents, Mn taken up by ingestion or inhalation is transported across the epithelial membrane via DMT1 [66]. If DMT1 orthologues are responsible for $\mathrm{Mn}$ uptake in C. elegans, the loss of function or down-regulation of these transporters should reduce Mn sensitivity. To determine if $s m f-1, s m f-2$ and $s m f-3$ are involved in Mn uptake and toxicity in the worm, we made use of available deletion-mutants for each of the three genes. Mutant smf1(eh5), smf-2(gk133) and smf-3(ok1035) strains were obtained from the Caenorhabditis Genetic Center (CGC). Under standard culture conditions, none of them displayed any obvious abnormal phenotype with respect to body morphology, development, growth, reproduction or behavior (data not shown). The smf-1 deletion $e h 5$ resulted in a truncated SMF-1 protein containing only the 6 first transmembrane domains and predicted to be devoid of Mn transport activity (Fig. 1B). When exposed to Mn, smf-1(eh5) mutants were twice as resistant as wild-type, with a $\mathrm{LD}_{50}=94 \mathrm{mM}$ (Fig. 4). The smf-3 deletion ok 1035 removes about $1,8 \mathrm{~kb}$ in a region encompassing exons 4 to 8 . It leads to a loss of at least two of the transmembrane domains 1 to 8 , preventing the resulting truncated protein from adopting its functional topology. In addition, the likely loss of TMD6 impairs divalent metal $\left(\mathrm{Me}^{2+}\right)$ transport as this domain was proven essential for $\mathrm{H}^{+} / \mathrm{Me}^{2+}$ symporter activity [47]. In support of this assumption, the smf-3(ok1035) mutant displayed the highest resistance to $\mathrm{Mn}$ exposure with a $\mathrm{LD}_{50}=126 \mathrm{mM}$ (Fig. 4). The hyper-resistance to Mn exposure exhibited by both smf-1(eh5) and smf-3(ok1035) mutants suggests that the Mn-induced toxicity observed in wild type worms was at least, in part, mediated through Mn uptake by the DMT1-like isoforms. A reasonable explanation for this observation is that Mn uptake in these mutants is impaired and that both SMF-3 and SMF-1 are required for normal and optimal Mn uptake.

The deletion mutant smf-2(gk133) is hypersensitive to Mn treatment. Concerning smf-2, the $g k 133$ deletion affects the last hundred bases of its promoter up to the third intron, which likely results in the production of a defective protein, lacking the $\mathrm{N}$-terminal sequence up to the $5^{\text {th }}$ TMD (Fig. 1B). Unlike smf-1(eh5) and smf-3(ok1035), smf-2(gk133) mutant display a significant hypersensitivity to $\mathrm{Mn}$ exposure with a $\mathrm{LD}_{50}=26 \mathrm{mM}$ (Fig. 4), suggesting a protective role for SMF-2 against Mn toxicity.

smf-3(ok1035) mutants take up less Mn while smf-2(gk133) mutants take up more. To confirm that the SMF proteins are involved in $\mathrm{Mn}$ uptake, following acute Mn exposure, we collected wild-type, smf1(eh5), smf-2(gk133), and smf-3(ok1035) worms, and processed them for Atomic Absorption Spectroscopy (AAS) analysis to measure their $\mathrm{Mn}$ content. All four strains showed dose-dependent increases in Mn content (Fig. 5A). smf-1(eh5) mutants accumulated less $\mathrm{Mn}$ than wildtype animals, but the trend was not significant at any of the tested doses. Strikingly, smf-2(gk133) accumulated 
A

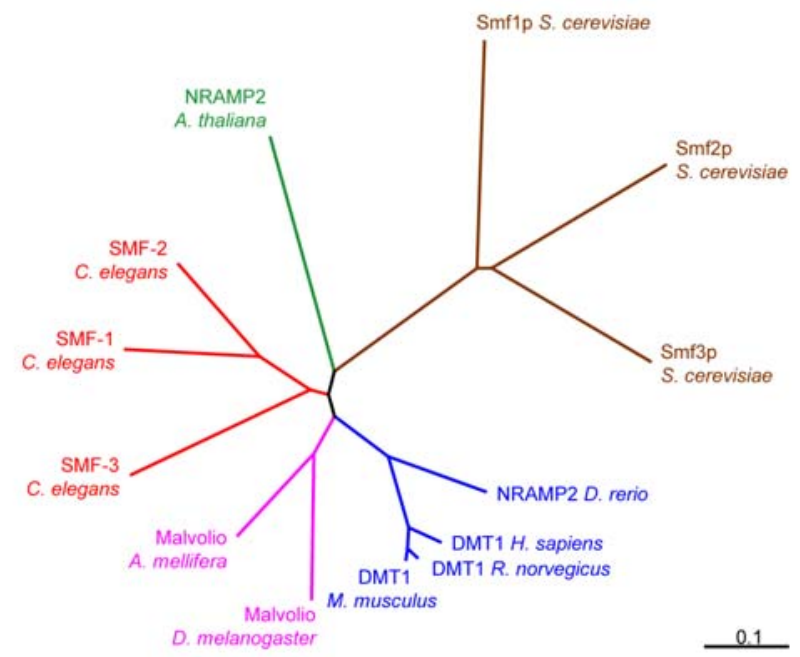

B
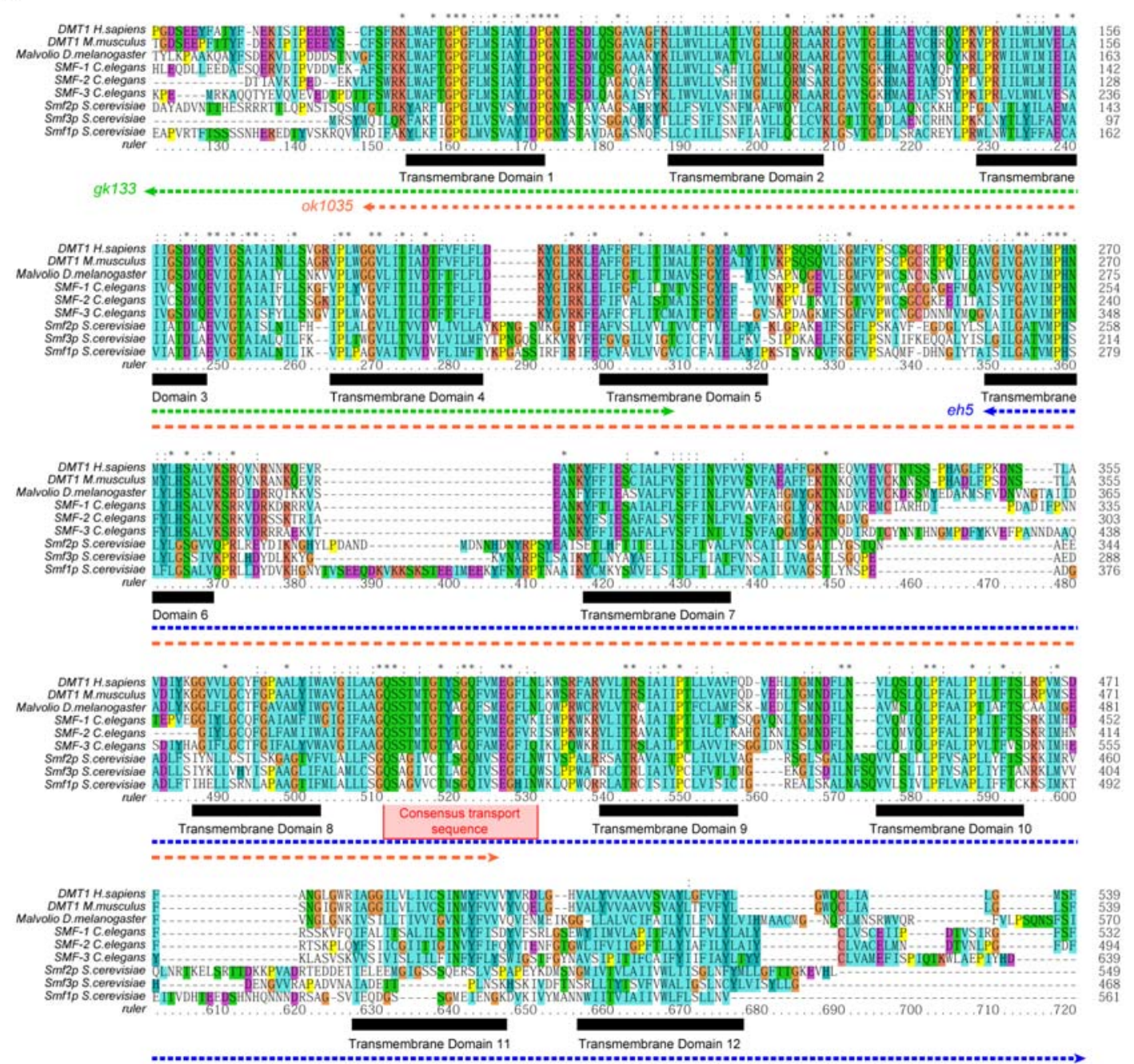

Figure 1. The $C$. elegans genome encodes 3 SMF transporters orthologous to the plant, fungi and animal DMT protein family. (A) Unrooted phylogenetic tree of a subset of eukaryotic members of the DMT family of transporters. C. elegans SMF proteins are more closely related to the animal than to the fungus or plant orthologues. (B) Multiple alignment of animal DMT1 orthologues. The 12-transmembrane domain topology of vertebrate DMT1 is conserved in C. elegans SMF proteins (black boxes), as well as the consensus transport sequence (red box). Dotted arrows indicate regions of the proteins affected by the deletion alleles eh5, gk113, and ok1035 of smf-1, 2, and 3 respectively. Amino acids with similar biochemical properties are highlighted with the same color. * represent residues conserved in all aligned sequences, : corresponds to highly conserved residues and. to less conserved residues.

doi:10.1371/journal.pone.0007792.g001 


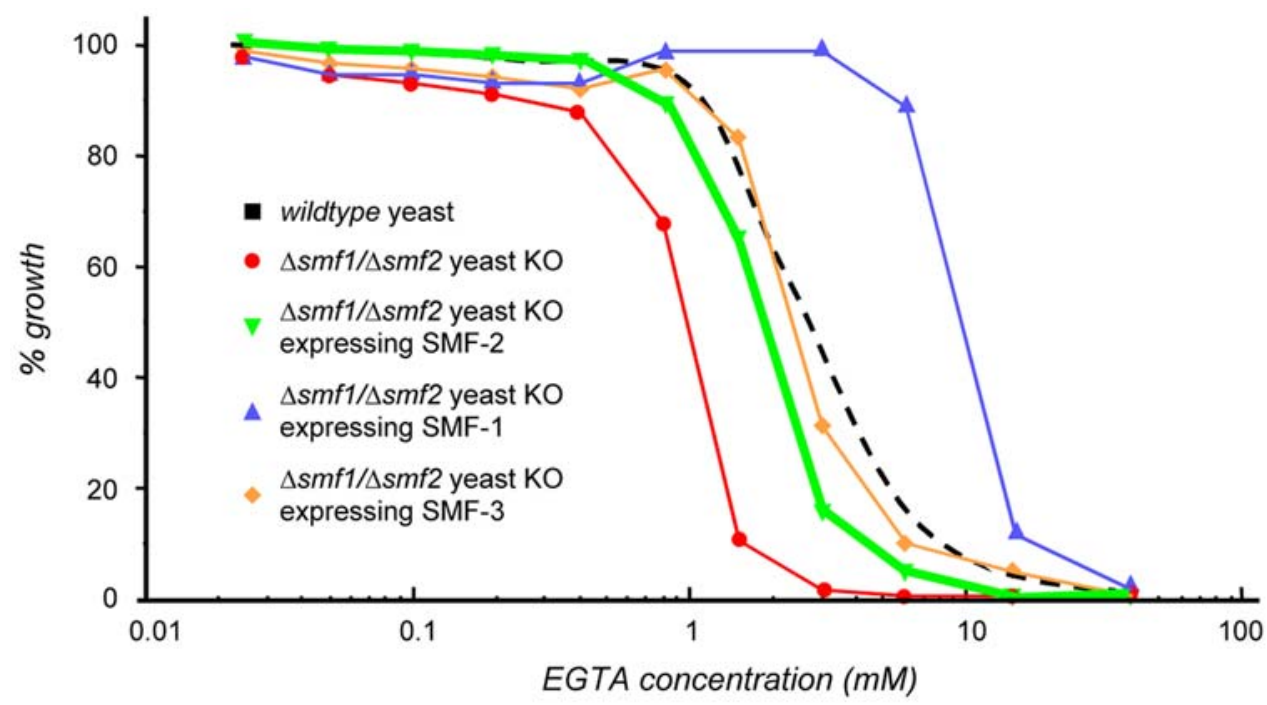

Figure 2. C. elegans SMF transporters rescue EGTA sensitivity of yeast $\Delta S m f 1+\Delta S m f-2$ mutant. S. cerevisiae double-mutant $S m f 1 \Delta+S m f-2 \Delta$ is hypersensitive to exposure to the divalent cation chelator EGTA (red), when compared to wildtype (dotted black line). Transvections of $C$. elegans smf-1 (blue), smf-2 (green) or smf-3 (orange) cDNA rescue the double-mutant hypersensitivity to EGTA. doi:10.1371/journal.pone.0007792.g002
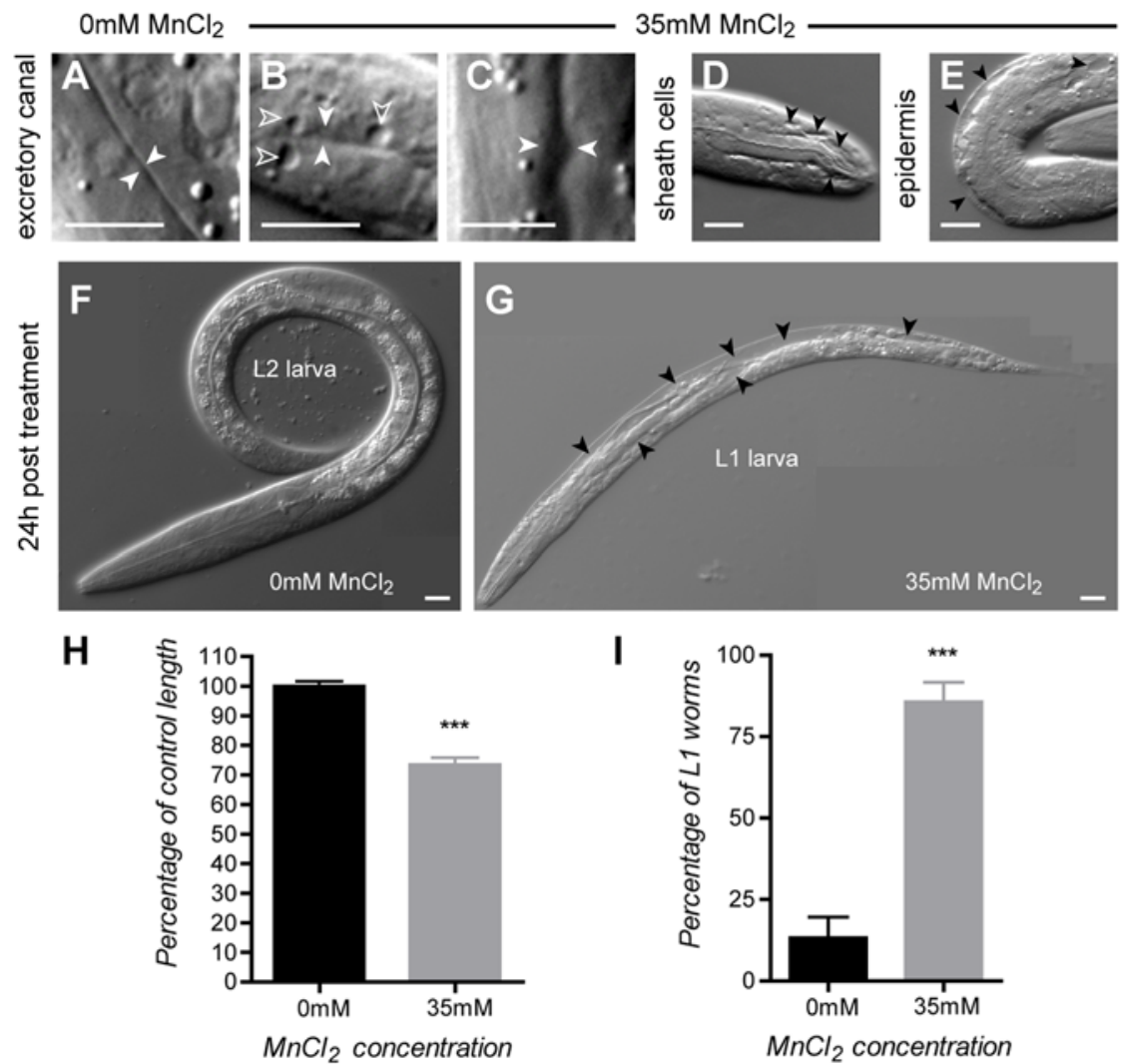

Figure 3. Mn exposure leads to severe osmoregulation defects and developmental delay. (A) Excretory canal in a control wild type L1 larva (solid white arrow heads). (B, C) Enlargement of the excretory canal in L1 larvae acutely exposed to $35 \mathrm{mM} \mathrm{MnCl}_{2}$, after $24 \mathrm{~h}$ (solid white arrowheads) is associated with vacuolization (hollow arrowheads). Vacuoles are also observed in the sheath cells of the chemosensory organs (D) and in the epidermis (E). (F) Control larva $24 \mathrm{~h}$ after $0 \mathrm{mM} \mathrm{MnCl}$, treatment. (G) Dying vacuolated (black arrowheads) larva $24 \mathrm{~h}$ after $35 \mathrm{mM} \mathrm{MnCl}_{2}$ exposure. (H) worms exposed to $35 \mathrm{mM} \mathrm{MnCl}_{2}$ (grey) are about $30 \%$ shorter than control animals (black). l, most larvae exposed to $35 \mathrm{mM} \mathrm{MnCl}$ are still in L1 stage at $24 \mathrm{~h}$ post-treatment when control animals are L2. Error bars represent SEM, ${ }^{* * *} \mathrm{p}<0.001$, scale bars are $5 \mu \mathrm{m}$. doi:10.1371/journal.pone.0007792.g003 


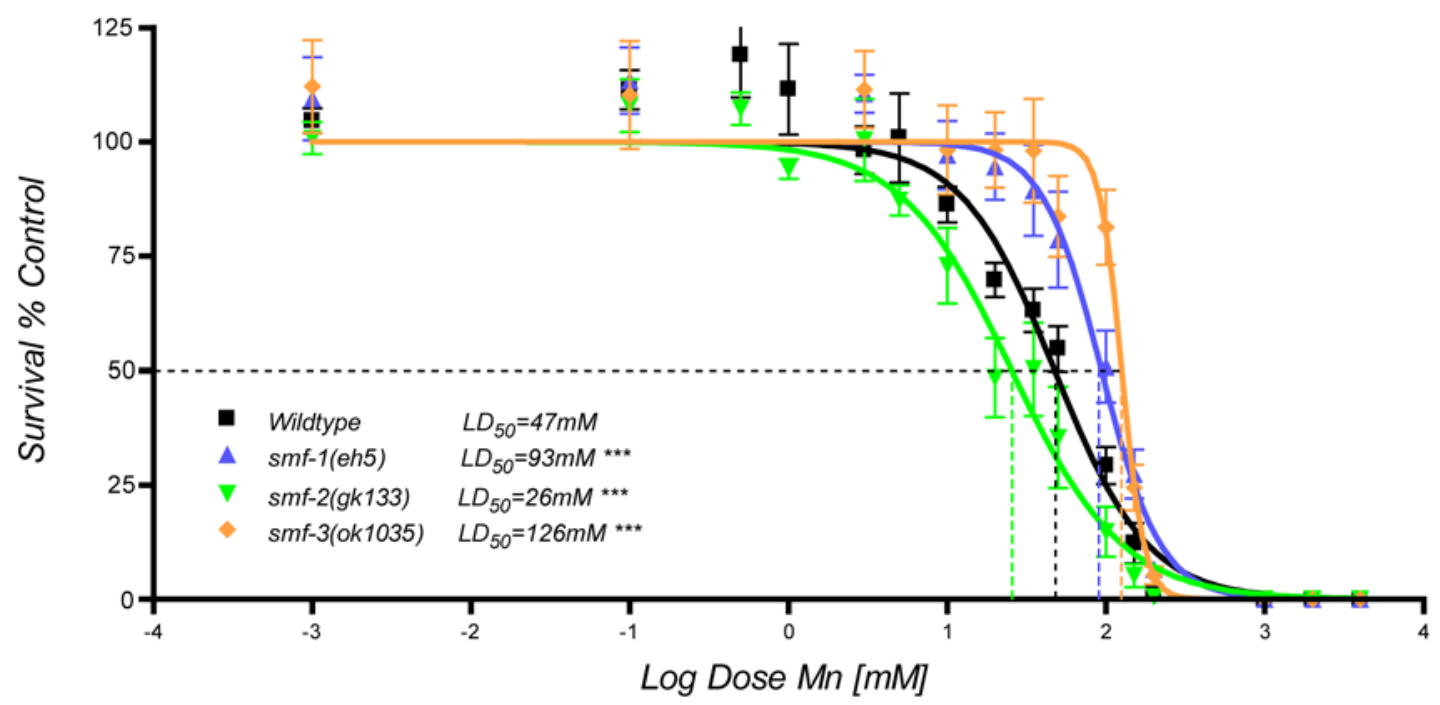

Figure 4. Dose-response lethality curves reveal a differential sensitivity to Mn exposure for smf mutants compared to wild type worms. Upon 30 min exposure to $\mathrm{MnCl}_{2}$ as $\mathrm{L} 1$ larvae the lethal concentration $50\left(\mathrm{LD}_{50}\right)$ at which half of the worms were dead at $24 \mathrm{~h}$, was $47 \mathrm{mM}$ for wildtype worms (black, $\mathrm{N}=12$ ), $93 \mathrm{mM}$ for smf-1 mutants (blue, $\mathrm{N}=6$ ), $26 \mathrm{mM}$ for smf-2 mutants (green, $\mathrm{N}=7$ ), and $126 \mathrm{mM}$ for smf-3 mutants (orange, $\mathrm{N}=5$ ). Error bars represent $\mathrm{SEM},{ }^{* * *} \mathrm{p}<0.001$. doi:10.1371/journal.pone.0007792.g004

significantly more $\mathrm{Mn}$ at $35 \mathrm{mM}(\mathrm{p}<0.05), 100 \mathrm{mM}(\mathrm{p}<0.001)$ and $150 \mathrm{mM}(\mathrm{p}<0.001)$ than any other strain, while $\operatorname{smf}-3(0 k 1035)$ mutants accumulated significantly less $\mathrm{Mn}$ than the other strains, both at $100 \mathrm{mM}(\mathrm{p}<0.001)$ and $150 \mathrm{mM}(\mathrm{p}<0.001)$.

smf-1(eh5) mutants exhibit a Mn-dependent decrease in Fe content while smf-2(gk133) mutants are naturally depleted in $\mathrm{Fe}$. In addition to $\mathrm{Mn}^{2+}$, DMT1 can transport a wide range of metallic cations including $\mathrm{Zn}^{2+}, \mathrm{Pb}^{2+}, \mathrm{Cd}^{2+}, \mathrm{Ni}^{2+}, \mathrm{Co}^{2+}$, and $\mathrm{Fe}^{2+}[67]$. It has been shown in rodents and cell cultures that $\mathrm{Mn}$ and $\mathrm{Fe}$ compete for transport via DMT1 $[66,68]$. In particular, high intracellular Fe levels lead to reduced $\mathrm{Mn}$ intake in rats [69]. To take into account a potential influence of Fe levels on Mn content, we analyzed Fe levels in parallel to Mn content. This analysis revealed that smf-2(gk133) mutants have a significantly lower $\mathrm{Fe}$ content than the other strains, which was not affected by Mn dose (Fig. 5B). Wildtype Fe levels were not significantly impacted by 0 to $100 \mathrm{mM} \mathrm{Mn}$ exposures, but showed an increase at $150 \mathrm{mM}$. Upon transient Mn depletion, smf-1(eh5) and smf-3(ok1035) mutants displayed higher Fe levels, but they underwent a gradual Fe depletion with increasing $\mathrm{Mn}$ doses (Fig. 5B). smf-1(eh5) Fe content significantly dropped below WT levels when exposed to $150 \mathrm{mM} \mathrm{Mn}$, suggesting a role for SMF-1 in Fe uptake at high Mn doses.

$S M F-1$ and SMF-3 expression partially overlaps in the main epithelia while $S M F-2$ is restricted to minor epithelial tissues.

To understand better the differential roles of the smf genes in $C$. elegans, we studied their expression pattern. We generated transgenic strains expressing the green fluorescent protein (GFP) under the control of smf-1, smf-2 or smf-3 promoters (smf-1::GFP, smf-2::GFP, smf-3::GFP), as well as strains expressing GFP-tagged SMF-1, SMF-2 and SMF-3 (SMF-1::GFP, SMF-2::GFP, SMF$3: \because G F P)$. In all cases the GFP signal was detected from late embryogenesis to adult stage, with generally higher expression levels in young larvae (Ll stage). None of the transgenic strains obtained showed any reproductive, developmental, behavioral or morphological defects, or intracellular GFP aggregates, thus establishing that the transgenes did not adversely affect the worms' physiology and that their expression levels were likely within the physiological range.
Both smf-1::GFP and SMF-1::GFP were prominently expressed in the anterior and posterior intestine and associated gland cells (Fig. 6A, 6B, 6C). A strong expression was observed in the anchor cell (AC) during larval life and in the adult proximal uterus resulting from the fusion of $\mathrm{AC}$ with uvl, uv2 and utse cells (Fig. 6A, 6D, 6E). GFP signal was also consistently seen in the adult spermatheca (Fig. 6F). Fainter expression was observed in the major epidermis hyp7, in the pharyngeal muscles, and in a subset of anterior sensory neurons, ring neurons, and posteriorhead neurons (Fig. 6A, 6G). SMF-3::GFP was mostly observed all along the intestine, with a weaker expression in the most proximal and distal regions (Fig. 6H, 6I). A weak epidermal expression in hyp 1-6, hyp7 and hyp8-12, and in head and tail neurons was also seen (Fig. 6J, 6K, 6L, 6M). In contrast to the broad SMF-1::GFP and SMF-3::GFP expression patterns, SMF-2::GFP was mostly restricted to the mc1, mc2, mc3 epithelial cells of the pharynx and the pharyngeal-intestinal valve cells vpil-6, displaying an anteriorposterior expression gradient (Fig. 6N, 6O). SMF-2 was also observed in the gonad sheath cells at the adult stage (Fig. 6P, 6Q).

$S M F-1$ and $S M F-3$ are apically localized while $S M F-2$ is mainly cytoplasmic. The full-length SMF-1::GFP and SMF-3::GFP allowed us to observe the intracellular localization of SMF-1 and SMF-3. In vertebrates, the DMT1 isoforms are mostly localized at the apical side in intestinal, rectal and kidney epithelial cells [66]. In agreement, SMF-1::GFP and SMF-3::GFP were localized at the apical plasma membrane in all epithelia in which they were expressed (Fig. 7A, 7B). Conversely, SMF-2::GFP was associated with intracellular cytoplasmic compartments and to a lesser extent to the apical membrane of the mc epithelial cells (Fig. 7C).

Variation in SMF protein levels/localization upon Mn exposure. Studies in vertebrates and yeast showed that DMT1 and DMT1-like isoforms are differentially regulated by metals, such as Fe and Mn [66]. In particular, modulation of expression and protein levels by Fe has been extensively studied [66]. In vertebrates, the DMT1 gene encodes two to four isoforms, defining two functional types which depend upon the presence of an Iron-Responsive Element (IRE) in their 3'UTR (+IRE isoform) or its absence (-IRE isoform) [70]. A consensus sequence for this IRE has been identified in 
A

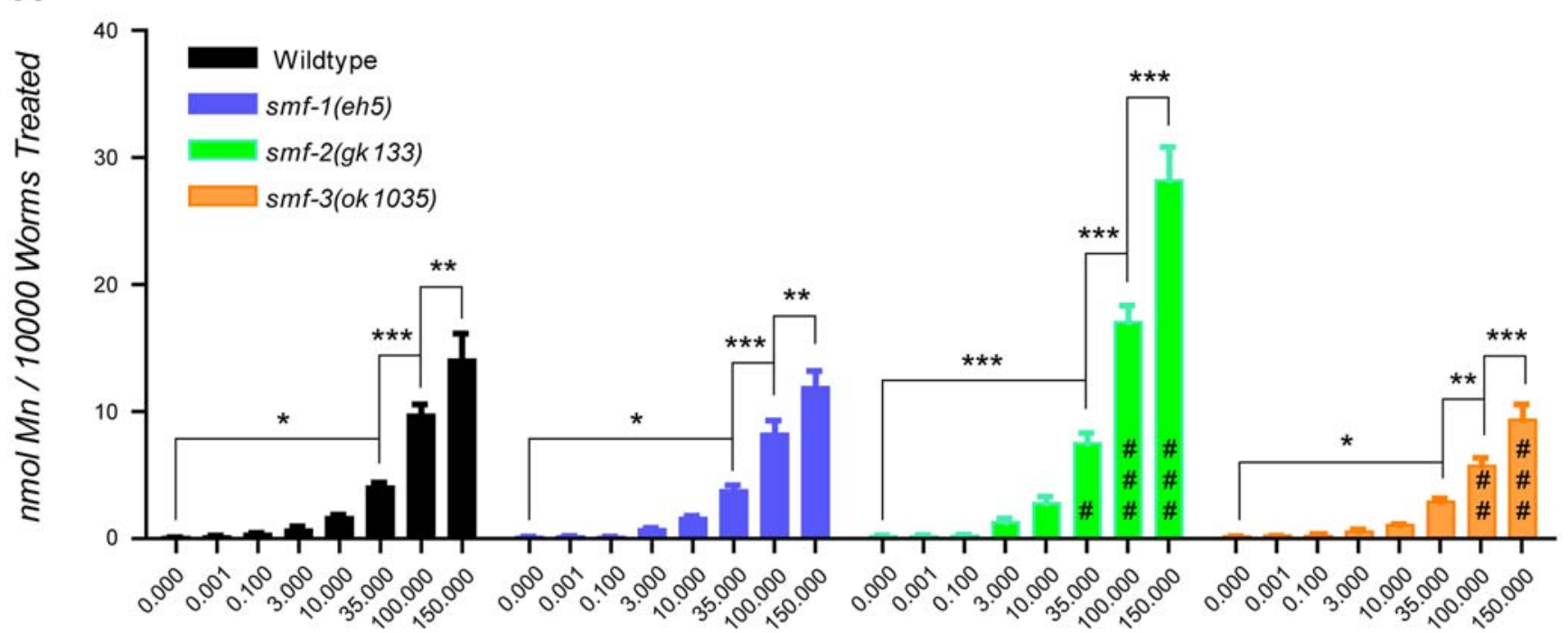

Mn Concentration ( $m M)$

B

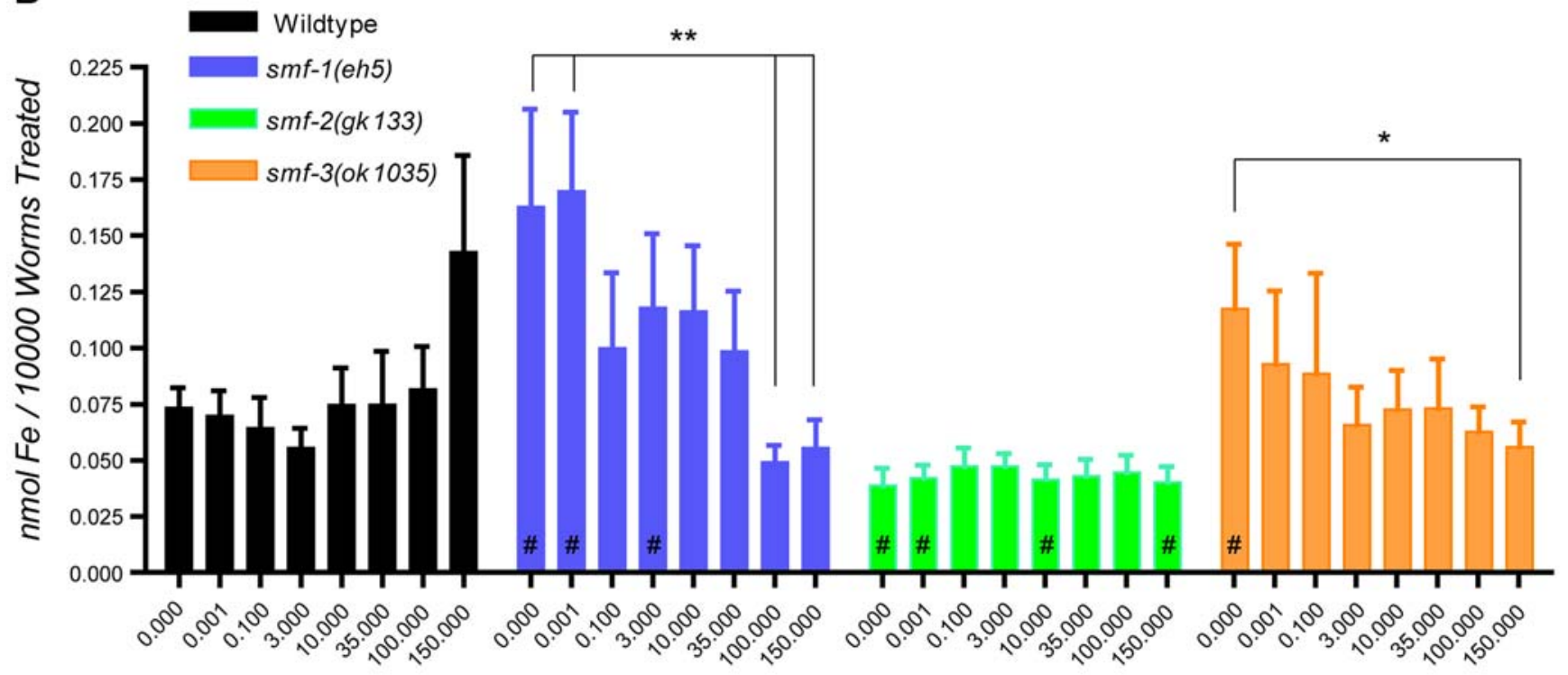

Mn Concentration ( $m M$ )

Figure 5. Variations in Mn and Fe content in smf mutant worms upon Mn exposure. (A) WT and smf mutants take up Mn in a dosedependent manner. smf-2(gk133) (green) takes up significantly more Mn than WT (black) and other mutant worms following exposure to 35 (\# $\mathrm{p}<0.05), 100$ (\#\# $\mathrm{p}<0.001)$ and $150 \mathrm{mM}$ (\#\#\# p<0.001) $\mathrm{MnCl}_{2}$. smf-3(ok1035) (orange) mutants take up significantly less Mn than other worms at 100 (\#\# $<<0.01$ ) and $150 \mathrm{mM}$ (\#\#\# $<<0.001$ ). (B) Fe content varies differentially in smf mutants and WT upon Mn exposure. smf-2(gk133) (green) display significantly lower Fe levels (\# $\mathbf{p}<0.05$ ), while smf-1(eh5) and smf-3(ok1035) mutants show higher Fe levels (\# $p<0.05)$ than WT in absence of $\mathrm{Mn}$ treatment $(0 \mathrm{mM})$, and at very low $\mathrm{Mn}$ concentration for smf-1(eh5) $(0.001 \mathrm{mM})$. Error bars represent SEM. While \# designate significant differences between genetic backgrounds exposed to the same manganese dose, ${ }^{*}$ indicate significant differences between exposure doses within the same C. elegans strain: $\# /^{*} p<0.05$. \#\#/** $p<0.01$, \#\#\#/***$p<0.001$.

doi:10.1371/journal.pone.0007792.g005

several proteins involved in Fe metabolism and regulation [71]. Yeast studies also showed differential regulation of $\operatorname{Smf}$ genes upon $\mathrm{Mn}$ and Fe exposure or depletion [55]. More specifically, in Mnreplete conditions, Smflp and Smf2p are targeted to the vacuole for degradation, but they accumulate at the plasma membrane and in intracellular vesicles upon Mn depletion [56,72,73]. Unlike Smflp and Smf2p, which are regulated by $\mathrm{Mn}$ at a post- translational level, Sm3p is regulated by $\mathrm{Fe}$ at a transcriptional level [56]. Given the impact of metal cation levels ( $\mathrm{Fe}$ and $\mathrm{Mn}$ ) on DMT 1 isoform expression and localization in both vertebrate and fungus models, we addressed the possibility that C. elegans SMF activity may also change with environmental $\mathrm{Mn}$ concentration. We used C. elegans strains transgenic for the SMF::GFP reporters to follow DMT1-like protein expression and localization changes 

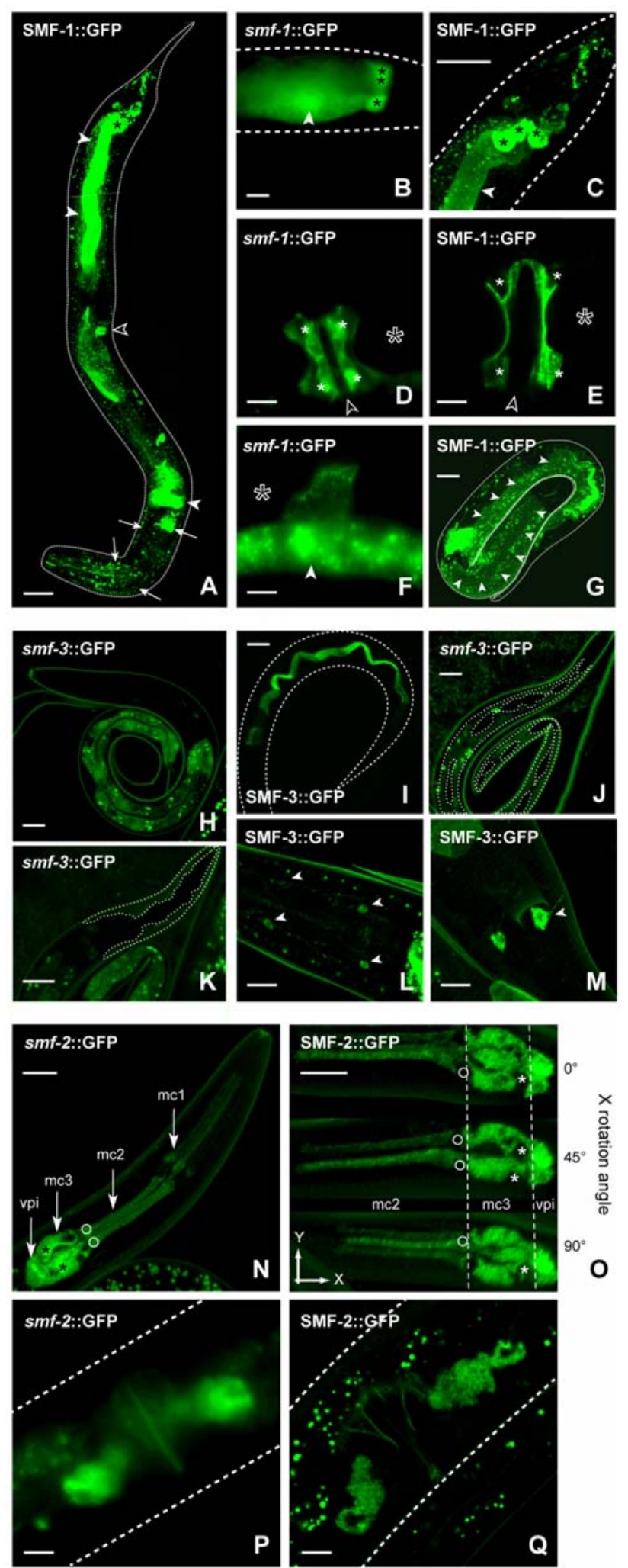

Figure 6. Expression pattern analysis of $C$. elegans smf genes. A, SMF-1::GFP strongly localizes to the anterior and posterior intestine (solid white arrowheads), to the anchor cell (hollow arrowhead) and to head neurons (white arrows). smf-1::GFP and SMF-1::GFP reveal expression of smf-1 gene in rectal gland cells (B,C, black asterisks), in the uterus (uv1, uv2, utse syncytium, D,E, solid white asterisks) as well as in the adult spermatheca $(F)$ and the $L 1$ hyp7 epidermis ( $G$, white arrowheads). Dotted lines outline the cuticle of the worm. Hollow asterisks indicate position of fertilized embryos. Hollow arrowheads indicate position of the vulva. smf-3 is mainly expressed in the intestine as revealed by smf-3::GFP $(\mathrm{H})$ and SMF-3::GFP (I), in the major epidermis hyp7 ( $\mathrm{J}$, dotted line) and head epidermis hyp1-6 ( $\mathrm{K}$, dotted line), and in head (L) and tail neurons (M). An antero-posterior gradient of smf-2 expression is noticeable in the 9 marginal epithelial cells of the pharynx $(\mathrm{mc} 1, \mathrm{mc} 2, \mathrm{mc} 3)$ and the 6 vpi cells of the pharyngeo-intestinal valve $(\mathrm{N}$ and $\mathrm{O})$. Fainter expression is consistently observed in the proximal gonad (P, Q). Scale bars are $5 \mu \mathrm{m}$.

doi:10.1371/journal.pone.0007792.g006

upon Mn exposure. We performed the same Ll acute treatment protocol as for our Mn toxicity and Mn content measurements, and collected worms at 1, 5 or 30 hours post-treatments for image analysis. SMF-1::GFP and SMF-2::GFP expressing worms did not show any obvious changes in either expression levels or intracellular localization. However, 1 hour post-treatment, SMF-3::GFP was translocated to apical vesicular compartments in the L1 intestine (Fig. 8A, 8B); at 5 hours the intestinal GFP signal was considerably reduced; it was not solely restricted to the apical plasma membrane, showing also cytoplasmic expression (Fig. 8C). This phenomenon was reversible, since at 30 hours post-exposure, both the expression levels and the subcellular localization of SMF-3::GFP returned to normal (Fig. 8D). These observations are supported by quantifications of SMF-3::GFP fluorescence at 5 and 30 hours, showing a significant decrease of SMF-3::GFP levels at 5 hours, but not at 30 hours post-treatment (Fig. 8E). This result suggests that SMF-3 function is regulated by Mn levels and further supports a major role for SMF-3 in Mn uptake.

Variation in smf gene expression by RT-PCR. Given the fact that $C$. elegans expresses DMT1-like isoforms with partially overlapping expression patterns, we examined if they displayed some functional redundancy and if their relative expression levels were dependent on each other and on metal levels. We also wondered whether SMF-3::GFP down-regulation upon Mn exposure could be explained by transcriptional inhibition. To address these issues we conducted quantitative real-time reverse-PGR assays (QRTPCR) on wild-type, smf-1(eh5), smf-2(gk133), and smf-3(ok1035) worms acutely exposed to $0,1,10,100 \mathrm{mM}$ of $\mathrm{MnCl}_{2}$. Both primers sets used to monitor smf gene transcriptional levels showed that smf-1 and smf-3 were up-regulated at $0.1 \mathrm{mM}$ of $\mathrm{Mn}$ and down-regulated at 10 and $100 \mathrm{mM} \mathrm{Mn}$ in WT and smf mutants, but not in smf-2(gk133) (Fig. 9A, 9B, 9G). As expected, smf-1

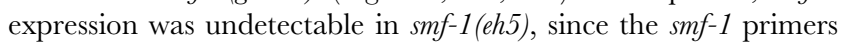
were chosen within the deleted region in $\operatorname{smf-1(eh5)~(Fig.~9A).~In~}$ $s m f-1(e h 5)$ and $s m f-3(o k 1035)$ mutants, smf-2 was also up-regulated at $0.1 \mathrm{mM}$ of $\mathrm{Mn}$ and down-regulated at 10 and $100 \mathrm{mM} \mathrm{Mn}$, while in WT, smf-2 expression was not affected by any of the Mn levels (Fig. 9B).

Noticeably, smf-2 was strongly overexpressed in smf-1(eh5) (Fig. 9B). In $s m f-3$ (ok1035), all $s m f$ genes were found overexpressed compared to WT, especially smf-3 (Fig. 9C), implying that a functional SMF-3 is required to maintain normally low smf gene expression. Lastly, smf gene expression was not influenced by $\mathrm{Mn}$ exposure in the smf-2(gk133) genetic background (Fig. 9A, 9B, 9C). This observation supports a role for SMF-2 in smf gene regulation upon changes in environmental Mn status.

\section{Discussion}

We identified three new functional members of the DMT1 family in C. elegans. All three rescued the yeast $\triangle S M F 1+2$ EGTA sensitivity, and revealed important for metal homeostasis in the worm, with very little functional overlap. SMF-1 and SMF-3 were 
SMF-1::GFP

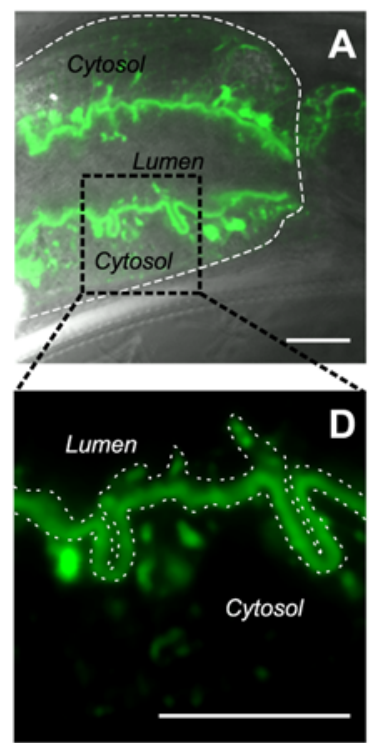

SMF-2::GFP

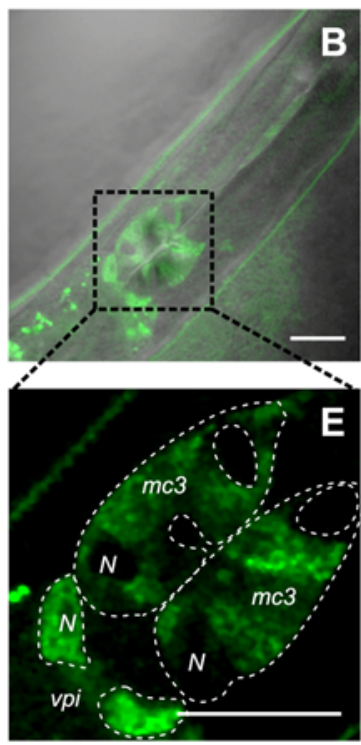

SMF-3::GFP

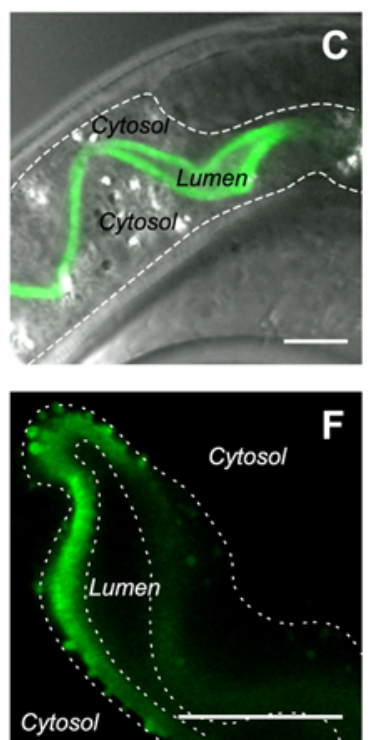

Figure 7. Subcellular localization of SMF::GFP reporters. SMF-1::GFP mostly localizes to the apical plasma membrane of the intestine and subapical compartments (A, D, dotted line in A underlines the basolateral membrane of the intestine, dotted lines in D delimit its apical plasma membrane). SMF-2::GFP is seen in cytoplasmic organelles in $\mathrm{mc}$ and vpi cells ( $\mathrm{E}$, cell plasma membranes are marked by a dotted line, $N$ indicate the position of the nuclei). SMF-3::GFP is mainly restricted to the apical plasma membrane of the intestine and apical vesicular organelles (C, F, dotted line in $\mathrm{C}$ underlines the basolateral membrane of the intestine, dotted lines in D delimit its apical plasma membrane). Scale bars are $5 \mu \mathrm{m}$. doi:10.1371/journal.pone.0007792.g007

broadly expressed in C. elegans epithelia; they were required for Mn uptake and toxicity, and exhibited the classical apical localization of DMT1. Conversely, SMF-2 expression was essentially restricted to specialized pharyngeal cells, where it was predominantly cytoplasmic, and to endow partial protection against Mn-induced toxicity. SMF-1 and SMF-2 were both required for normal Fe intake, SMF-2 being necessary for Mndependent Fe content regulation. Mn exposure revealed influential on transcriptional regulation of the three smf genes, but only affected the post-translational regulation of SMF-3. Our results
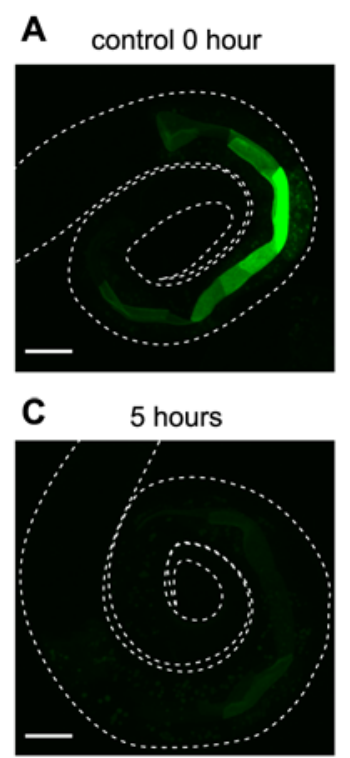
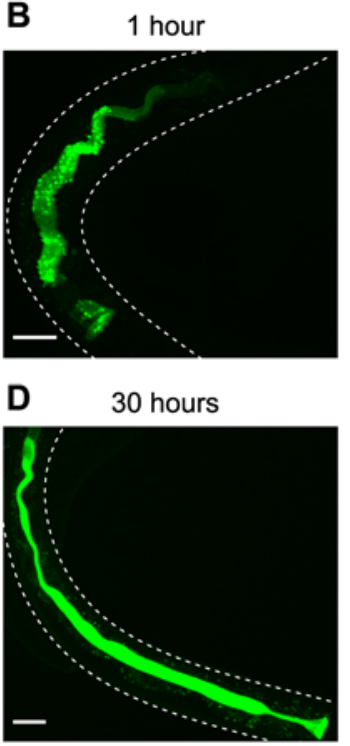

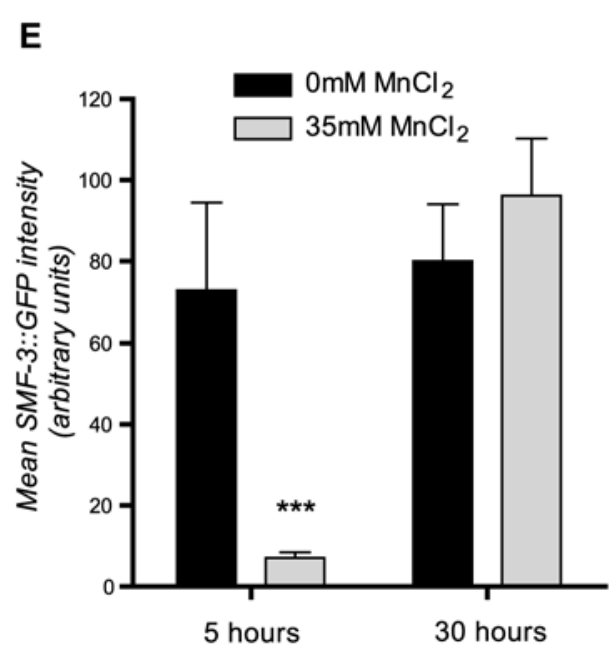

5 hours

30 hours

Figure 8. SMF-3::GFP is down-regulated upon Mn exposure. SMF-3::GFP signal is strongly detected at the apical plasma membrane prior Mn treatment (A). After 1 hour of exposure to $35 \mathrm{mM} \mathrm{MnCl2}$, SMF-3-GFP localizes to sub-apical vesicular compartments (B). C, SMF-3::GFP signal is strongly decreased at 5 hours post-treatment. After a day of recovery, SMF-3::GFP expression returns to control levels and SMF-3::GFP relocates to the apical plasma membrane (D). Scale bars are $5 \mu \mathrm{m}$. (E) quantification of apical plasma-membrane SMF-3::GFP in the whole intestine after 5 and $30 \mathrm{~h}$ of exposure in control and treated animals. While \# designate significant differences between genetic backgrounds exposed to the same manganese dose, ${ }^{*}$ indicate significant differences between exposure doses within the same $C$. elegans strain: $\# /{ }^{*} p<0.05$. $\# \# / /^{* *} \mathrm{p}<0.01, \# \# \# / p<0.001$. doi:10.1371/journal.pone.0007792.g008 

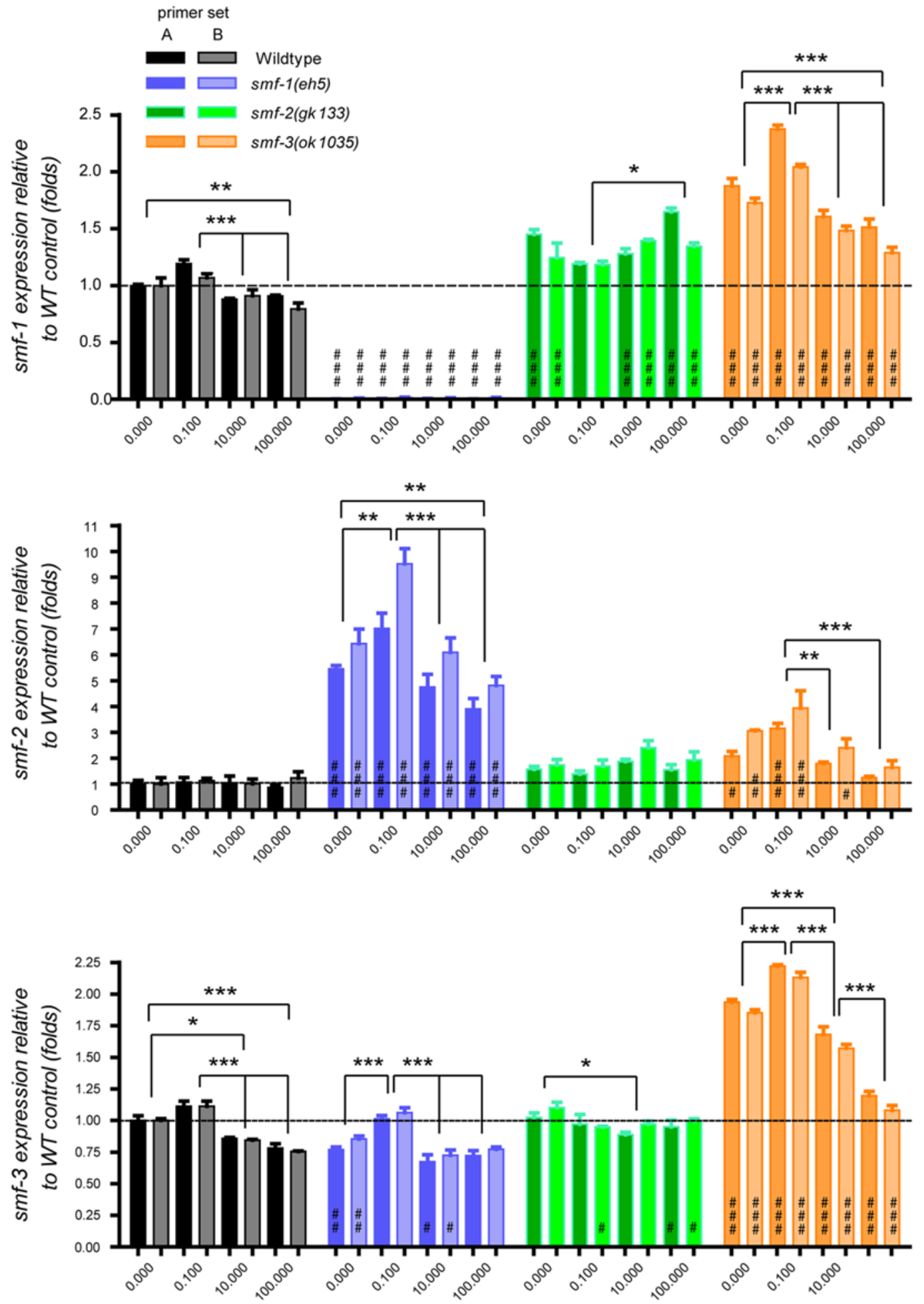

Mn Concentration ( $m M$ )

Figure 9. Genotype and Mn exposure influence on $s m f$ gene expression. Both independent primer sets corresponding to the $3^{\prime}$ end of the cDNA and used to assess smf gene expression (set A bright colors, and set B faded colors) give consistent results (A, B, C). smf-1 and smf-3 mRNA levels display similar variations, increasing at low $\mathrm{Mn}$ exposure $(0.1 \mathrm{mM})$ and decreasing upon high $\mathrm{Mn}$ concentrations (10 $\mathrm{mM}$ and $100 \mathrm{mM})$ in WT, smf-1 and smf-3 mutants (A, C, black, blue, orange). smf-2 mRNA levels follow the same tendency in smf-1 and smf-3 mutants but are not affected by $\mathrm{Mn}$ exposure in WT (B). Independent of Mn exposure, smf-1(eh5) mutant is characterized by a strong up-regulation of smf-2 expression (B, blue). Compared to other genotypes, smf expression in smf-2(gk133) does not appear to correlate with Mn exposure (A, B, C, green). The smf-3(ok1035) mutant exhibits higher smf gene expression levels regardless of $\mathrm{Mn}$ exposure dose (A, B, C, orange). While \# designate significant differences between genetic backgrounds exposed to the same manganese dose, ${ }^{*}$ indicate significant differences between exposure doses within the same $C$. elegans strain: $\# /^{*} p<0.05$. \#\#/** $\mathrm{p}<0.01, \# \# \# / p<0.001$. Displayed significance levels between treatments reflect the weakest significance score obtained between both primer sets.

doi:10.1371/journal.pone.0007792.g009 
unravel a complex metal-regulation system involving 3 distinct DMTs coordinately regulated by metal levels, and bear interesting implications on the modulation of the vertebrate DMT activities carried out by NRAMP1 and DMT1 isoforms.

$S M F-3$ regulates and is specifically regulated by $M n$. In addition to expression and localization data, Mn content measurement and dose-response lethality curves in $s m f$ mutants clearly indicate that SMF-3 is a major Mn transporter in the worm, which is required for Mn-induced toxicity. smf-3(ok1035) is the mutant most resistant to acute Mn exposure (Fig. 4). SMF-3 is expressed in the epidermis and located all along the intestine, where most $\mathrm{Mn}$ absorption likely occurs (Fig. 6). SMF-3 sub-cellular localization is apical, consistent with a role in Mn uptake (Fig. 7). smf-3(ok1035) is the only mutant that displays a significant decrease in $\mathrm{Mn}$ content upon $\mathrm{MnCl}_{2}$ treatment (Fig. 5), implying that SMF-1 or SMF-2 cannot compensate for SMF-3 depletion. Moreover, SMF-3 levels and apical localization are strongly decreased 5 hours after toxic $\mathrm{Mn}$ exposure and are restored after a 24 hour recovery period (Fig. 8). Finally, QRT-PCR revealed a decrease in smf-3 mRNA levels upon $\mathrm{Mn}$ exposure at 10 and $100 \mathrm{mM}$ (Fig. 9). These observations support the notion that $s m f-3$ is down-regulated both transcriptionally and post-translationally upon Mn exposure, which limits toxic accumulation of $\mathrm{Mn}$ in the worm. After a day of recovery allowing for the excretion of $\mathrm{Mn}$ accumulated upon previous exposure, SMF-3 levels return back to normal levels, permitting nutritional divalent cation uptake. Our experiments support a regulation of SMF-3 levels at the post-translational level, by relocating and degrading SMF-3. The translocation of SMF-3 likely to endosomal compartments in response to excessive $\mathrm{Mn}$ exposure can be related to the vacuolar targeting of Smflp and Smf2p in Mn-replete conditions in yeast $[56,73,74]$. In porcine kidney cells, (-IRE)-DMT1 isoforms have been shown to be more rapidly and efficiently internalized upon metal exposure when compared to (+IRE)-DMT1 isoforms [39]. Although we did not find any typical IRE sequence in any of the three smf $3^{\prime} \mathrm{UTR}$ sequences (data not shown), we observed a drastic internalization of SMF-3 upon Mn treatment, but not of SMF-1 or SMF-2. Additionally, DMT1 has the highest affinity for $\mathrm{Mn}^{2+}$ [67] and SMF-3 is the C. elegans orthologue that is responsible for most Mn uptake. Hence, in C. elegans, SMF-3 may be the functional counterpart of the vertebrate -IRE forms of DMT1.

An atypical role for $S M F-2$ in Mn homeostasis. smf-2 is located downstream of $\operatorname{smf}-1$ on the $\mathrm{X}$ chromosome. Due to the absence of any splice acceptor site in the smf-2 sequence and based on our GFP-reporter analysis, it is unlikely that $s m f-1$ and $s m f-2$ belong to the same operon. Indeed, both exhibited very distinct expression patterns (Fig. 6) and mutations in smf-1 or smf-2 have opposite effects on Mn sensitivity (Fig. 2). The high degree of conservation between both genes suggests that they result from a duplication that happened early in the Caenorhabditis lineage, since it is also found on the C. briggsae and C. remanei X chromosomes (http://dev. wormbase.org/db/seq/gbrowse_syn/pecan/?search_src = elegans; name $=$ chrX\%3A6716267..6721098). smf-2(gk133) mutants exhibited hypersensitivity to Mn exposure and a higher Mn content than wild type worms (Fig. 2, 5), indicating that SMF-2 partially protects against $\mathrm{Mn}$ exposure, which may not be solely explained by a role in Mn uptake. The intestinal expression of SMF-1 and SMF-3 from late embryogenesis to adulthood strongly supports their involvement in metal uptake, while SMF-2 expression and subcellular localization in the pharyngeal epithelial cells is intriguing, since these cells are not known to be involved in nutrient or toxicant uptake (http://www. wormatlas.org/handbook/alimentary/alimentaryl.htm).

SMF-2 may rather be involved in the regulation of Mn toxicity, either by allowing its excretion, its sequestration, or the modulation of Mn uptake via the other DMT1-like isoforms, or a combination of the above. Because of the structural and functional similarities between SMF-3, SMF-1 and SMF-2, we favor the idea that pharyngeal SMF-2 takes up metallic cations, allowing for the overall regulation of $\mathrm{Mn}$ content. Several hypotheses can be formulated. First, SMF-2 could work as a sensor of environmental $\mathrm{Mn}$ levels, and a downstream signaling pathway would either impact Mn uptake via SMF-3 and SMF-1 or $\mathrm{Mn}$ excretion via as of yet uncharacterized transporters. The anterior-posterior gradient of SMF-2 expression in the pharynx would provide a differential mechanism to accurately estimate metal concentrations. Second, SMF-2-driven inhibition of $\mathrm{Mn}$ uptake could involve modulation of the pharyngeal pumping. The marginal cells of the pharynx, which express SMF-2, are electrically coupled to the pharyngeal muscles by gap-junctions [75]. In mammalian cells, high Mn concentrations have been shown to impact cardiomyocyte contractility by inhibiting the $\mathrm{Ca}^{2+}$-ATPase [76,77]. Considering the structural and functional similarities between the C. elegans pharynx and the vertebrate heart [78], Mn uptake via SMF-2 in marginal cells might impact pharyngeal muscle activity. Decrease in pharyngeal pumping would result in reduced nutrient and $\mathrm{Mn}$ intake. This could also account for the slow growth of Mn-treated animals. Third, our Fe content analysis suggests that SMF-2 is an important $\mathrm{Fe}$ transporter. The lack of SMF-2 leads to lower Fe levels in the smf-2(gk133) worms (Fig. 5B), which would allow for increased Mn uptake by SMF-3 or SMF-1 (Fig. 5A). Moreover, Fe content is high at low $\mathrm{Mn}$ exposure, and drops at high Mn exposure in smf1 (eh5) and smf-3(ok1035) mutants, while it is constant in smf2 (gk133) mutants (Fig. 5B). The smf gene expression also follows this trend. It suggests that SMF-2 is required for Mn-dependent variations in $\mathrm{Fe}$ content and $s m f$ gene expression. SMF-2 is active in a different cell type as SMF-1 and SMF-3 (Fig. 4), and those cells are connected through gap-junctions. To reconcile these data, we propose that SMF-2-dependent Fe-uptake is inhibited at high Mn doses, leading to a Fe-depletion in the pharynx and a reversed Fe gradient across the animal. In turn this Fe imbalance, together with increased Mn content would lead to the down-regulation of smf genes in a SMF-2-dependent manner (Fig. 10B).

Based on SMF-2::GFP subcellular localization (Fig. 7), which appears to be in puncta in the cytoplasm, but not in the nucleus, SMF-2 could be present in most membranous intracellular organelles of the endo-lysosomal pathway. Its activity could be increased by the V-ATPase-driven acidification of those compartments, similarly to DMT1 in endosomes and NRAMP1 in phagosomes [33], leading to metal transport to the cytosol. On the one hand, generation of double-transgenic C. elegans strains to conduct colocalization studies with lyzosomal (LAMP-1 and LAMP-2) and endosomal (RAB-5, RAB-7) markers would be beneficial in determining if the puncta seen in Fig. 7 correspond to endo-lyzosomal compartments. On the other hand, colocalization with an essential V-ATPase subunit, such as VHA-8 [89], and observation of orange acridine ( $\mathrm{pH}$-sensitive vital dye) staining in SMF-2-positive puncta and measurement of C. elegans Fe content upon variation in environmental $\mathrm{pH}$, could be used to study the $\mathrm{pH}$-dependency of SMF-2 function.

SMF-1: minor in Mn physiology, major in Fe homeostasis? Though expression pattern, sub-cellular localization and mutant analysis might suggest an important role for SMF-1 in $\mathrm{Mn}^{2+}$ uptake, $\mathrm{Mn}$ content measurements failed to show a significant difference between wild type and smf-1(eh5) mutant worms. Thus, a difference in Mn uptake may not account for their resistance to Mn exposure. Several hypotheses may be advanced to explain this apparent discrepancy. First, SMF-1 may mediate Mn toxicity 


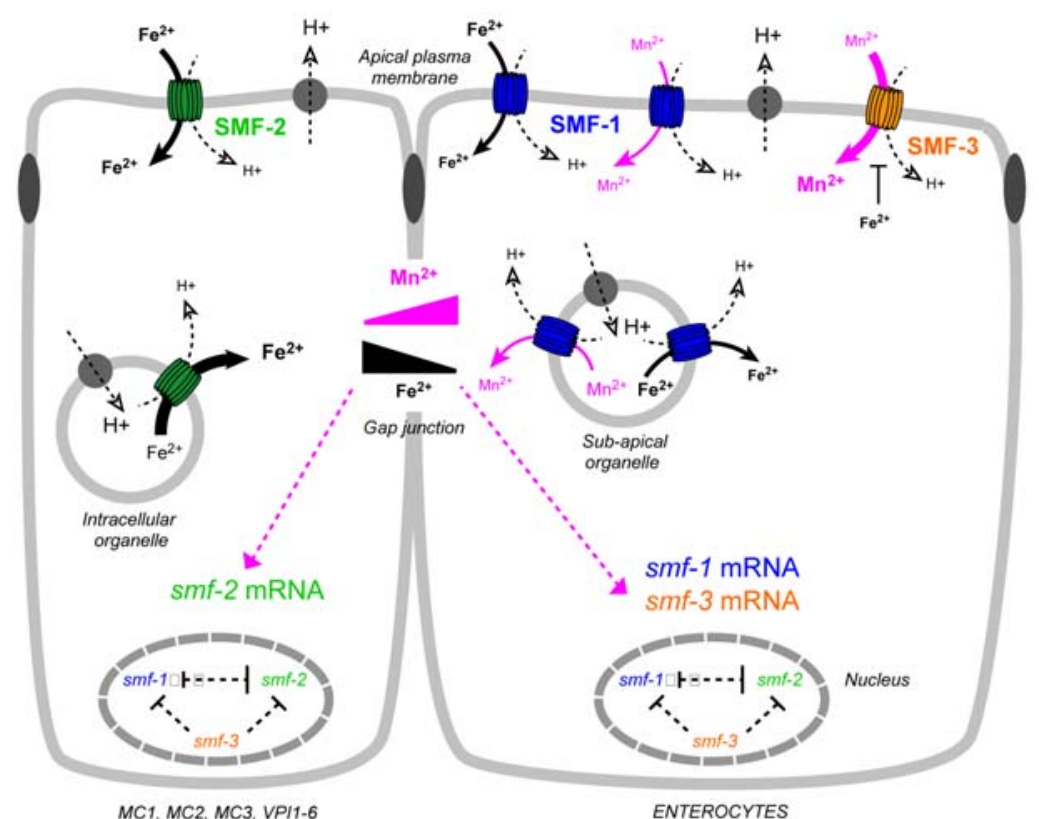

\section{B High Mn exposure}

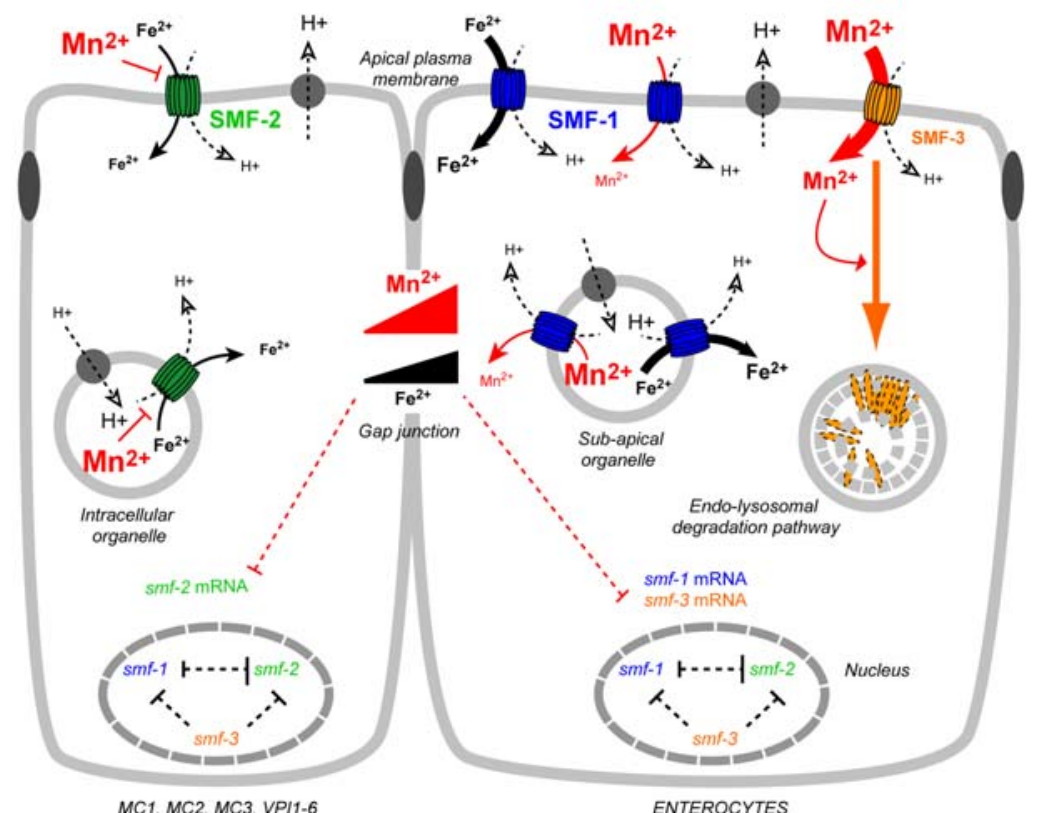

Figure 10. Working model for Mn and Fe uptake by SMF transporters in C. elegans. A: Regulation of Mn, Fe contents and SMF transporters upon low Mn exposure (0.001 $\mathrm{mM}$ to $3 \mathrm{mM}$ ), which is believed to be beneficial for the worm physiology [92]. B: Regulation of Mn, Fe contents and SMF transporters upon high $\mathrm{Mn}$ exposure $(50 \mathrm{mM}$ to $150 \mathrm{mM}$ ), which was shown to be toxic (Fig. 3, 4). We propose that SMF-3* is the main transporter responsible for $\mathrm{Mn}$ uptake (A), and that it is degraded upon exposure to high $\mathrm{Mn}$ concentrations (B). Since high Fe content limit $\mathrm{Mn}$ uptake, SMF-3 may be inhibited by intracellular Fe (A). SMF-1 would be involved in Mn uptake to a lesser extent, and together with SMF-2*, would be responsible for Fe uptake. Upon Low Mn exposure SMF-2 would be mostly required for Fe uptake (A), whereas upon high Mn exposure, SMF-2 would be inhibited and SMF-1 would partially compensate for Fe uptake (B). In the case of SMF-2 and SMF-1, metal uptake could essentially take place in acidified endosomal compartments, as SMF-2 is mainly cytoplasmic and SMF-1 is detected in sub-apical compartments. Gap-junction communications between pharyngeal epithelia, vpi cells and intestinal cells permit $\mathrm{Mn}^{2+}$ and $\mathrm{Fe}^{2+}$ to diffuse distant from their site of uptake, allowing metal-dependent regulation of $s m f$ mRNA stability or transcription. A prediction of our model is that the Fe gradient established by SMF-2 activity would be reversed upon high $\mathrm{Mn}$ exposure (B), and could constitute the signal for $s m f$ expression regulation. Since basal smf mRNA levels depend on the integrity of each smf genomic sequence (Fig. 9), transcriptions of smf genes are assumed to be interdependent, maybe because they require a common transcription factor. * SMF-2 might transport Mn and SMF-3 might transport Fe, but these possibilities are not explored in this model. The size of the text reflects the concentration of the species.

doi:10.1371/journal.pone.0007792.g010 
subsequent to Mn uptake at the apical membrane of the intestine, transporting Mn from endocytic compartments to the cytosol. In absence of SMF-1, endocytic Mn content would remain the same, but $\mathrm{Mn}$ would not be transported across the membrane to reach the cytosol and trigger toxicity (Fig. 10). The presence of SMF-1 in intracellular vesicular organelles supports this idea (Fig. 7). When located at the apical plasma membrane, SMF-1 would then be largely inactive; being activated upon acidification of the endocytic vesicles by the vacuolar-ATPase $[33,48,79]$. Second, SMF-1's contribution to Mn uptake may be minor compared to SMF-3 and $\mathrm{Mn}$ content measurements would not be able to reflect the relatively limited, yet physiologically significant variations in $\mathrm{Mn}$ content. This alternative explanation is logical within the context of the DMT family, in which different isoforms have been shown to transport distinct cations with variable efficiencies. Hence, the vertebrate NRAMP1 is a critical Fe transporter in macrophages $[80,81,82]$, and has a poor affinity for Mn compared to DMT1 [67]. SMF-1 may thus be the counterpart of NRAMP1 in the worm, being predominantly required for Fe uptake. Additionally, smf-1(eh5) is the only mutant which showed a significant Mndependent depletion of $\mathrm{Fe}$ content, despite the overexpression of $s m f-2$ in this context. It indicates that SMF-1 is required to take up or conserve $\mathrm{Fe}$ at high $\mathrm{Mn}$ concentrations, and may be responsible for Fe homeostasis (Fig. 10). Third, the QRT-PCR experiments

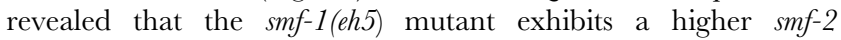
expression level than wildtype, smf-2(gk133) and smf-3(ok1035) worms. If as mentioned previously SMF-2 is protective against Mn toxicity, the resistant phenotype of the smf-1(eh5) mutant may reflect the overexpression of SMF-2. In this case, SMF-1 would not necessarily be involved in Mn transport. Fourth, the previous hypotheses are not mutually exclusive. Lastly, SMF-1 is able to rescue EGTA sensitivity in yeast beyond WT level (Fig. 2). This better rescue could be due to a higher transgene copy number or a better stability of the SMF-1 product in the yeast, resulting in higher SMF-1 levels and a hypermorphic phenotype. However, it could also indicate that this transporter has a broader range of substrates than SMF-2 and SMF-3 and may be functionally closer to a canonical DMT.

New insights for the study of the eukaryotic family of DMT1-related transporters. Our analysis of the C. elegans DMTs reveals that they are closely related structurally and functionally to their vertebrate orthologues. SMF-1 and SMF-3 are apically localized in epidermal and intestinal cells, consistent with observations about DMT1 distribution and sub-cellular localization in vertebrates $[47,85,86]$. Moreover, DMT1 proteins transport divalent metallic cations via coupling with the proton gradient [47] in part generated by the vacuolar-ATPase apically localized both in vertebrates and in $C$. elegans epithelia [66,89,90]. Interestingly, the three $C$. elegans isoforms display distinct expression levels and contribute differentially to $\mathrm{Mn}$ and Fe physiology, similarly to the +IRE, -IRE forms of DMT1 and NRAMP1 in vertebrates. The fact that SMF-2 antagonizes SMF-3 could be connected to the suspected antiporter activity of NRAMP1 relatively to the symporter activity of DMT1. NRAMP1 was proposed to be extruding metals against the proton gradient [91]. This activity would rely on specific residues located before the transmembrane domains (TMD) 9 and 12, which differ between NRAMP1 and DMT1. In particular, in mammals, NRAMP1 exhibits a basic residue K389 instead of a polar residue N403 for DMT1, at the end of the CTS. Interestingly, our multiple-alignment reveals that all other DMT1 orthologues including Malvolio, SMF-3 as well as yeast Smflp, Smf2p and Smf3p display a polar residue $(\mathrm{N}$ or $\mathrm{Q})$ at this position, while SMF1 and SMF-2 show a basic residue ( $\mathrm{R}$ or $\mathrm{K}$ ) similarly to NRAMP1 (Fig. 1). SMF-2 also seemed to limit Mn accumulation. However, unlike NRAMP1, SMF-1 and SMF-2 efficiently rescue the yeast $\triangle S M F 1+2$ EGTA sensitivity. Moreover, their expression in the proximal digestive system argues against an antiporter activity that would result in metal extrusion. Therefore, it would be very interesting to test the ability of SMF-1 and SMF-2 to rescue the heavy metal ion stress in $\triangle B S D 2 / \triangle R E R 1$ yeast mutants. Ultimately the symporter or antiporter activity of DMTs may depend on the metal ions and their concentrations on both sides of the membrane. Environmental, cytosolic and organelle metal concentrations in yeast, oocytes, macrophages or distinct $C$. elegans cell types may vary enough for DMTs to function differently depending on the context. Another point is that the specific expression patterns of SMF-1, SMF-2 and SMF-3 remained the same through the worm's post-embryonic life, implying that the cell-specific expression levels of smf genes are embryonically determined, further variations resulting from changes in metal exposure and physiology.

Analysis of our results allows us to propose a working model for $\mathrm{Mn}$ and $\mathrm{Fe}$ uptake regulation in C. elegans (Fig. 10). We favor the notion that metal ion gradients play the key role in the regulation of expression, sub-cellular localization and activity of DMTs in $C$. elegans. To further test this idea, fluorescent ferrochromes could be used to visualize in vivo the $\mathrm{Fe}$ gradient upon various $\mathrm{Mn}$ exposures. To yield further information on the metal-transport abilities of the various DMTs from worm to human, one could perform a systematical comparative analysis of DMT family member biochemical properties in yeast using complementation rescue of $\triangle B S D 2 / \triangle R E R 1$ and $\triangle S M F 1+2$ mutants, coupled to an in vivo study using metal-exposed transgenic $C$. elegans expressing tissue-specific chimerical DMT transgenes. Such a dual approach should help solve the paradox concerning the antiporter activity of NRAMP1 orthologues, and the effect of metal gradients on DMT activity.

To dissect out the relative contributions of each $s m f$ gene in metal physiology in C. elegans, the generation of double or triple smf mutants would also be of great interest. However generation of the $s m f-1 ; s m f-2$ double mutant and the triple mutant smf-1;smf-2;smf-3 would require a new deletion mutant in which both $s m f-1$ and $s m f-$ 2 expressions are lost, since the short genetic distance between both genes would make recombination events between smf-1(eh5) and $s m f-2(g k 133)$ very unlikely. In addition, considering that $s m f-2$

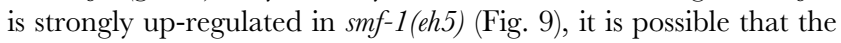
eh 5 deletion, by bringing smf- 1 promoting or enhancing sequences closer to the smf-2 gene, affects its natural expression. This potential issue should be carefully considered before further usage of $\operatorname{smf}-1($ eh5) to generate double-mutants. Combining RNAi and single mutants could be another way to address the redundancy issue, with the limitation that RNAi may not be fully penetrant or specific enough to affect single isoforms.

Relevance of our $\mathrm{C}$. elegans model to study Mn toxicity and related diseases. $\mathrm{Mn}$ exposure has long been suspected to be responsible for IPD cases. The commonalities between manganism and PD suggest that both pathologies, at least in part, rely on shared genetic networks and molecular mechanisms. Due to the increasing incidence of PD, notably in most populous countries [23], there is growing interest in identifying contributing genetic and environmental factors. In this context, development of amenable genetic models to study geneenvironment interactions is essential. Our work corroborates the utility of C. elegans as an appropriate complementary whole-animal model to decipher molecular mechanisms and genetic predisposition to metal induced toxicity. First, we showed that $C$. elegans takes up $\mathrm{Mn}$ readily and is sensitive to $\mathrm{Mn}$ exposure, exhibiting developmental delay, excretory cell and osmoregulatory defects which can be related to kidney and bladder defects observed in 
rodents [83,84] and man [44]. The high Mn concentrations used in our study (when compared to cell-culture studies in which the $\mathrm{LD}_{50}$ approximates $1 \mathrm{mM}$ for $24 \mathrm{~h}$ exposures) likely reflect three characteristics of the model. C. elegans is sheathed with a cuticle impermeable to ions and only few orifices would physically allow metal ion uptake, namely the mouth, the rectum, the pore cell of the excretory system and the chemosensory organs. In support of this, the Mn content measured by atomic absorption spetrophotometry (AAS) in wild-type worms treated at the $\mathrm{LD}_{50}(47 \mathrm{mM})$ corresponded to an effective dose of $12.5 \mathrm{mM}$. Another point to consider is that the worms in the present study were acutely treated (30 min) in contrast to most cell culture studies that involve more protracted exposures $(>24 \mathrm{~h})$. It is also likely that the worm, naturally living in soil, where ion concentrations fluctuate greatly and rapidly, may be physiologically more robust than mammalian cells. Second, our functional analysis of the SMF transporters in the yeast (Fig. 2) together with the metal content measurements in wild type and smf mutant worms (Fig. 5A), show that the three C. elegans DMTs are involved in metal uptake regulation. Third, two of these transporters, SMF-1 and SMF-3 are mostly expressed in the intestine, a portal for $\mathrm{Mn}$ and Fe uptake both in worms and vertebrates. SMF-1 and SMF-3 are also apically localized in epidermal and intestinal cells, consistent with observations about DMT1 distribution and sub-cellular localization in vertebrates $[47,85,86]$. Fourth, several facts inherent to the regulation of DMTs by $\mathrm{Mn}$ or $\mathrm{Fe}$ and vice-versa previously described in mammalian models also hold true in C. elegans. For example, we showed that low Mn exposures $(0.1 \mathrm{mM})$ tend to increase and high concentrations $(100 \mathrm{mM})$ to decrease $\operatorname{smf}$ gene expression (Fig. 9). SMF-2 was required in this regulation process as $\mathrm{Mn}$ concentration-dependent effects are not observed in the $s m f-2$ (gk133) mutant (Fig. 5, 9). Fifth, $\mathrm{Fe}$ and $\mathrm{Mn}$ tissue levels are interdependent and depend on environmental concentrations. In the smf-2(gk133) mutant, low $\mathrm{Fe}$ content is associated with an increased ability to take up Mn. In smf1(eh5), Fe content decreases with increasing Mn exposure (Fig. 5). Last, we found that SMF-3 N-terminal sequence contains a putative Mitochondrial Targeting Sequence (MTS, probability 93.66\% given by MitoProt, http://ihg2.helmholtz-muenchen.de/ihg/

\section{References}

1. Post JE (1999) Manganese oxide minerals: crystal structures and economic and environmental significance. Proc Natl Acad Sci U S A 96: 3447-3454.

2. Saric M (1986) Bronchial hyperreactivity and occupational asthma. Am J Ind Med 9: 217-219.

3. Wedler FC, Denman RB (1984) Glutamine synthetase: the major Mn(II) enzyme in mammalian brain. Curr Top Cell Regul 24: 153-169.

4. Addess KJ, Basilion JP, Klausner RD, Rouault TA, Pardi A (1997) Structure and dynamics of the iron responsive element RNA: implications for binding of the RNA by iron regulatory binding proteins. J Mol Biol 274: 7283.

5. Aschner M, Gannon M, Kimelberg HK (1992) Manganese uptake and efflux in cultured rat astrocytes. J Neurochem 58: 730-735.

6. Fitsanakis VA, Aschner M (2005) The importance of glutamate, glycine, and gamma-aminobutyric acid transport and regulation in manganese, mercury and lead neurotoxicity. Toxicol Appl Pharmacol 204: 343-354.

7. Liao SL, Chen CJ (2001) Manganese stimulates stellation of cultured rat cortical astrocytes. Neuroreport 12: 3877-3881.

8. Malecki EA, Devenyi AG, Beard JL, Connor JR (1999) Existing and emerging mechanisms for transport of iron and manganese to the brain. J Neurosci Res 56: 113-122.

9. Prohaska JR (1987) Functions of trace elements in brain metabolism. Physiol Rev 67: 858-901.

10. Takeda A, Sotogaku N, Oku N (2003) Influence of manganese on the release of neurotransmitters in rat striatum. Brain Res 965: 279-282.

11. Aschner M (2000) Manganese: brain transport and emerging research needs. Environ Health Perspect 108 Suppl 3: 429-432.

12. Aschner M (2002) Open issues from the 15th International Conference on Manganese. Neurotoxicology 23: 123-125.

13. Aschner M, Aschner JL (1991) Manganese neurotoxicity: cellular effects and blood-brain barrier transport. Neurosci Biobehav Rev 15: 333-340. mitoprot.html): MPRVHRQSRWNSVSFSGFFLQISGIKPRF. Our analysis of SMF-3::GFP sub-cellular localization did not reveal any epithelial mitochondrial targeting in vivo. However, it could happen in tissues that were not investigated in this study such as neurons, or in situations that were not covered by our experimental conditions. If it was confirmed, a mitochondrial targeting of DMT1 orthologues would mean that their activity could directly impact mitochondrial function and potentially apoptosis, in particular in neurodegenerative processes $[87,88]$.

Taken together, our data suggest that Mn uptake and toxicity mechanisms involving DMTs are conserved from nematodes to man. Because other genetically amenable invertebrates such as $D$. melanogatser only express one DMT (Malvolio, Mvl), and given the large panel of currently available techniques on the nematode (toxicology, molecular biology, forward and reverse genetics, confocal and electron-microscopy, biochemistry), C. elegans provides a convenient in vivo platform to further investigate metal toxicity and related neurodegenerative disorders in which DMT1related transporters are suspected to play an important role $[87,88]$.

\section{Acknowledgments}

We thank Philippe Gros, Greg Govoni and Karine Botturi for their contributions to early parts of this project. We thank Yuji Kohara for smf-1, smf-2 and smf-3 cDNA, the Caenorhabditis Genetic Center (CGC, Minnesota, USA) for providing $C$. elegans strains, the International $C$. elegans Knockout Consortium for generation of alleles gk133 (C. elegans Reverse Genetics Core Facility at UBC, www.celeganskoconsortium.omrf.org) and ok1035 (C. elegans Gene Knockout Project at OMRF, www.mutantfactory.ouhsc. edu) and Sanger Centre Knockout Service for the generation of the allele eh5

\section{Author Contributions}

Conceived and designed the experiments: AB JJE MA. Performed the experiments: $\mathrm{CA} \mathrm{AB} \mathrm{AL}$. Analyzed the data: $\mathrm{CA}$ AB KME JJE MA. Contributed reagents/materials/analysis tools: JGA KME. Wrote the paper: $\mathrm{CA} \mathrm{AB}$ MA.
14. Thompson K, Molina RM, Donaghey T, Schwob JE, Brain JD, et al. (2007) Olfactory uptake of manganese requires DMT1 and is enhanced by anemia. Faseb J 21: 223-230.

15. Dorman DC, McManus BE, Parkinson CU, Manuel CA, McElveen AM, et al. (2004) Nasal toxicity of manganese sulfate and manganese phosphate in young male rats following subchronic (13-week) inhalation exposure. Inhal Toxicol 16: 481-488.

16. Calne DB, Chu NS, Huang CC, Lu CS, Olanow W (1994) Manganism and idiopathic parkinsonism: similarities and differences. Neurology 44: 1583-1586.

17. Cersosimo MG, Koller WC (2006) The diagnosis of manganese-induced parkinsonism. Neurotoxicology 27: 340-346.

18. Olanow CW (2004) Manganese-induced parkinsonism and Parkinson's disease. Ann N Y Acad Sci 1012: 209-223.

19. Pfeifer GD, Roper JM, Dorman D, Lynam DR (2004) Health and environmental testing of manganese exhaust products from use of methylcyclopentadienyl manganese tricarbonyl in gasoline. Sci Total Environ 334-335: 397-408.

20. Ressler T, Wong J, Roos J (1999) Manganese speciation in exhaust particulates of automobiles using MMT-containing gasoline. J Synchrotron Radiat 6: 656-658.

21. Rollin H, Mathee A, Levin J, Theodorou P, Wewers F (2005) Blood manganese concentrations among first-grade schoolchildren in two South African cities. Environ Res 97: 93-99.

22. Brown RC, Lockwood AH, Sonawane BR (2005) Neurodegenerative diseases: an overview of environmental risk factors. Environ Health Perspect 113: $1250-1256$.

23. Dorsey ER, Constantinescu R, Thompson JP, Biglan KM, Holloway RG, et al. (2007) Projected number of people with Parkinson disease in the most populous nations, 2005 through 2030. Neurology 68: 384-386.

24. Mayeux R (2003) Epidemiology of neurodegeneration. Annu Rev Neurosci 26: 81-104. 
25. Murphy VA, Wadhwani KC, Smith QR, Rapoport SI (1991) Saturable transport of manganese(II) across the rat blood-brain barrier. J Neurochem 57: 948-954.

26. Aschner M, Gannon M (1994) Manganese (Mn) transport across the rat bloodbrain barrier: saturable and transferrin-dependent transport mechanisms. Brain Res Bull 33: 345-349.

27. Rabin O, Hegedus L, Bourre JM, Smith QR (1993) Rapid brain uptake of manganese(II) across the blood-brain barrier. J Neurochem 61: 509-517.

28. Andrews NC (1999) The iron transporter DMT1. Int J Biochem Cell Biol 31: 991-994.

29. Gruenheid S, Cellier M, Vidal S, Gros P (1995) Identification and characterization of a second mouse Nramp gene. Genomics 25: 514-525.

30. Vidal S, Belouchi AM, Cellier M, Beatty B, Gros P (1995) Cloning and characterization of a second human NRAMP gene on chromosome 12q13. Mamm Genome 6: 224-230.

31. Garrick MD, Dolan KG, Horbinski C, Ghio AJ, Higgins D, et al. (2003) DMT1: a mammalian transporter for multiple metals. Biometals 16: 41-54.

32. Gunshin H, Mackenzie B, Berger UV, Gunshin Y, Romero MF, et al. (1997) Cloning and characterization of a mammalian proton-coupled metal-ion transporter. Nature 388: 482-488.

33. Forbes JR, Gros P (2003) Iron, manganese, and cobalt transport by Nrampl (Slc1 la1) and Nramp2 (Slc1 la2) expressed at the plasma membrane. Blood 102: $1884-1892$.

34. Knopfel M, Zhao L, Garrick MD (2005) Transport of divalent transition-metal ions is lost in small-intestinal tissue of b/b Belgrade rats. Biochemistry 44: 3454-3465.

35. Cellier M, Prive G, Belouchi A, Kwan T, Rodrigues V, et al. (1995) Nramp defines a family of membrane proteins. Proc Natl Acad Sci U S A 92: 10089-10093.

36. Su MA, Trenor CC, Fleming JC, Fleming MD, Andrews NC (1998) The G185R mutation disrupts function of the iron transporter Nramp2. Blood 92: 2157-2163.

37. Beaumont C, Delaunay J, Hetet G, Grandchamp B, de Montalembert M, et al. (2006) Two new human DMT1 gene mutations in a patient with microcytic anemia, low ferritinemia, and liver iron overload. Blood 107: 4168-4170.

38. Iolascon A, d'Apolito M, Servedio V, Cimmino F, Piga A, et al. (2006) Microcytic anemia and hepatic iron overload in a child with compound heterozygous mutations in DMT1 (SCL11A2). Blood 107: 349-354.

39. Lam-Yuk-Tseung S, Camaschella C, Iolascon A, Gros P (2006) A novel R416C mutation in human DMT1 (SLC11A2) displays pleiotropic effects on function and causes microcytic anemia and hepatic iron overload. Blood Cells Mol Dis 36: $347-354$

40. Lam-Yuk-Tseung S, Mathieu M, Gros P (2005) Functional characterization of the E399D DMT1/NRAMP2/SLC11A2 protein produced by an exon 12 mutation in a patient with microcytic anemia and iron overload. Blood Cells Mol Dis 35: 212-216.

41. Mims MP, Prchal JT (2005) Divalent metal transporter 1. Hematology 10: 339-345.

42. Priwitzerova M, Nie G, Sheftel AD, Pospisilova D, Divoky V, et al. (2005) Functional consequences of the human DMT1 (SLC11A2) mutation on protein expression and iron uptake. Blood 106: 3985-3987.

43. Lee PL, Gelbart T, West C, Halloran C, Beutler E (1998) The human Nramp2 gene: characterization of the gene structure, alternative splicing, promoter region and polymorphisms. Blood Cells Mol Dis 24: 199-215.

44. Huang WH, Lin JL (2004) Acute renal failure following ingestion of manganesecontaining fertilizer. J Toxicol Clin Toxicol 42: 305-307.

45. Canonne-Hergaux F, Gruenheid S, Ponka P, Gros P (1999) Cellular and subcellular localization of the Nramp2 iron transporter in the intestinal brush border and regulation by dietary iron. Blood 93: 4406-4417.

46. Fleming MD, Andrews NC (1998) Mammalian iron transport: an unexpected link between metal homeostasis and host defense. J Lab Clin Med 132: 464 - 468.

47. Courville P, Chaloupka R, Cellier MF (2006) Recent progress in structurefunction analyses of Nramp proton-dependent metal-ion transporters. Biochem Cell Biol 84: 960-978.

48. Tabuchi M, Yoshimori T, Yamaguchi K, Yoshida T, Kishi F (2000) Human NRAMP2/DMT1, which mediates iron transport across endosomal membranes, is localized to late endosomes and lysosomes in HEp-2 cells. J Biol Chem 275: 22220-22228.

49. Gruenheid S, Canonne-Hergaux F, Gauthier S, Hackam DJ, Grinstein S, et al. (1999) The iron transport protein NRAMP2 is an integral membrane glycoprotein that colocalizes with transferrin in recycling endosomes. J Exp Med 189: 831-841.

50. Kannurpatti SS, Joshi PG, Joshi NB (2000) Calcium sequestering ability of mitochondria modulates influx of calcium through glutamate receptor channel. Neurochem Res 25: 1527-1536.

51. Touret N, Furuya W, Forbes J, Gros P, Grinstein S (2003) Dynamic traffic through the recycling compartment couples the metal transporter Nramp2 (DMT1) with the transferrin receptor. J Biol Chem 278: 25548-25557.

52. Eide DJ (1998) The molecular biology of metal ion transport in Saccharomyces cerevisiae. Annu Rev Nutr 18: 441-469.

53. Supek F, Supekova L, Nelson H, Nelson N (1996) A yeast manganese transporter related to the macrophage protein involved in conferring resistance to mycobacteria. Proc Natl Acad Sci U S A 93: 5105-5110.
54. Cohen A, Nelson H, Nelson N (2000) The family of SMF metal ion transporters in yeast cells. J Biol Chem 275: 33388-33394.

55. Portnoy ME, Jensen LT, Culotta VC (2002) The distinct methods by which manganese and iron regulate the Nramp transporters in yeast. Biochem J 362: $119-124$.

56. Portnoy ME, Liu XF, Culotta VC (2000) Saccharomyces cerevisiae expresses three functionally distinct homologues of the nramp family of metal transporters. Mol Cell Biol 20: 7893-7902.

57. Luk EE, Culotta VC (2001) Manganese superoxide dismutase in Saccharomyces cerevisiae acquires its metal co-factor through a pathway involving the Nramp metal transporter, Smf2p. J Biol Chem 276: 47556-47562.

58. Pinner E, Gruenheid S, Raymond M, Gros P (1997) Functional complementation of the yeast divalent cation transporter family SMF by NRAMP2, a member of the mammalian natural resistance-associated macrophage protein family. J Biol Chem 272: 28933-28938.

59. Thompson JD, Gibson TJ, Plewniak F, Jeanmougin F, Higgins DG (1997) The CLUSTAL_X windows interface: flexible strategies for multiple sequence alignment aided by quality analysis tools. Nucleic Acids Res 25: 4876-4882.

60. Thompson JD, Higgins DG, Gibson TJ (1994) CLUSTAL W: improving the sensitivity of progressive multiple sequence alignment through sequence weighting, position-specific gap penalties and weight matrix choice. Nucleic Acids Res 22: 4673-4680.

61. Brenner S (1974) The genetics of Caenorhabditis elegans. Genetics 77: 71-94.

62. Mello CC, Kramer JM, Stinchcomb D, Ambros V (1991) Efficient gene transfer in C.elegans: extrachromosomal maintenance and integration of transforming sequences. Embo J 10: 3959-3970.

63. Hoogewijs D, Houthoofd K, Matthijssens F, Vandesompele J, Vanfleteren JR (2008) Selection and validation of a set of reliable reference genes for quantitative sod gene expression analysis in C. elegans. BMC Mol Biol 9: 9.

64. Liegeois S, Benedetto A, Michaux G, Belliard G, Labouesse M (2007) Genes required for osmoregulation and apical secretion in Caenorhabditis elegans. Genetics 175: 709-724.

65. Nelson FK, Riddle DL (1984) Functional study of the Caenorhabditis elegans secretory-excretory system using laser microsurgery. J Exp Zool 231: 45-56.

66. Au C, Benedetto A, Aschner M (2008) Manganese transport in eukaryotes: the role of DMT1. Neurotoxicology 29: 569-576.

67. Garrick MD, Singleton ST, Vargas F, Kuo HC, Zhao L, et al. (2006) DMT1: which metals does it transport? Biol Res 39: 79-85.

68. Roth JA, Feng L, Dolan KG, Lis A, Garrick MD (2002) Effect of the iron chelator desferrioxamine on manganese-induced toxicity of rat pheochromocytoma (PC12) cells. J Neurosci Res 68: 76-83.

69. Thompson K, Molina R, Donaghey T, Brain JD, Wessling-Resnick M (2006) The influence of high iron diet on rat lung manganese absorption. Toxicol Appl Pharmacol 210: 17-23.

70. Mackenzie B, Takanaga H, Hubert N, Rolfs A, Hediger MA (2007) Functional properties of multiple isoforms of human divalent metal-ion transporter 1 (DMT1). Biochem J 403: 59-69.

71. Piccinelli P, Samuelsson T (2007) Evolution of the iron-responsive element. Rna 13: 952-966.

72. Eguez L, Chung YS, Kuchibhatla A, Paidhungat M, Garrett S (2004) Yeast $\mathrm{Mn} 2+$ transporter, Smflp, is regulated by ubiquitin-dependent vacuolar protein sorting. Genetics 167: 107-117.

73. Liu XF, Culotta VC (1999) Post-translation control of Nramp metal transport in yeast. Role of metal ions and the BSD2 gene. J Biol Chem 274: 4863-4868.

74. Jensen LT, Ajua-Alemanji M, Culotta VC (2003) The Saccharomyces cerevisiae high affinity phosphate transporter encoded by PHO84 also functions in manganese homeostasis. J Biol Chem 278: 42036-42040.

75. Albertson DG, Thomson JN (1976) The pharynx of Caenorhabditis elegans. Philos Trans R Soc Lond B Biol Sci 275: 299-325.

76. Bansal SK, Murthy RC, Chandra SV (1985) The effects of some divalent metals on cardiac and branchial Ca2+-ATPase in a freshwater fish Saccobranchus fossilis. Ecotoxicol Environ Saf 9: 373-377.

77. Steinberg SF, Chow YK, Bilezikian JP (1986) Regulation of rat heart membrane adenylate cyclase by magnesium and manganese. J Pharmacol Exp Ther 237: $764-772$.

78. Mango SE (2007) The G. elegans pharynx: a model for organogenesis. WormBook. pp 1-26.

79. Burdo JR, Menzies SL, Simpson IA, Garrick LM, Garrick MD, et al. (2001) Distribution of divalent metal transporter 1 and metal transport protein 1 in the normal and Belgrade rat. J Neurosci Res 66: 1198-1207.

80. Canonne-Hergaux F, Gruenheid S, Govoni G, Gros P (1999) The Nrampl protein and its role in resistance to infection and macrophage function. Proc Assoc Am Physicians 111: 283-289.

81. Mulero V, Searle S, Blackwell JM, BrockJH (2002) Solute carrier 11a1 (Slc1 la1; formerly Nramp1) regulates metabolism and release of iron acquired by phagocytic, but not transferrin-receptor-mediated, iron uptake. Biochem J 363: 89-94.

82. Searle S, Bright NA, Roach TI, Atkinson PG, Barton CH, et al. (1998) Localisation of Nrampl in macrophages: modulation with activation and infection. J Cell Sci 111(Pt 19): 2855-2866.

83. Guven K, Deveci E, Akba O, Onen A, de Pomerai D (1998) The accumulation and histological effects of organometallic fungicides Propineb and Maneb in the kidneys of fetus and female rats during pregnancy. Toxicol Lett 99: 91-98. 
84. Ponnapakkam T, Iszard M, Henry-Sam G (2003) Effects of oral administration of manganese on the kidneys and urinary bladder of Sprague-Dawley rats. Int J Toxicol 22: 227-232.

85. Canonne-Hergaux F, Gros P (2002) Expression of the iron transporter DMT1 in kidney from normal and anemic mk mice. Kidney Int 62: 147-156.

86. Mackenzie B, Garrick MD (2005) Iron Imports. II. Iron uptake at the apical membrane in the intestine. Am J Physiol Gastrointest Liver Physiol 289: G981-986.

87. Salazar J, Mena N, Hunot S, Prigent A, Alvarez-Fischer D, et al. (2008) Divalent metal transporter 1 (DMT1) contributes to neurodegeneration in animal models of Parkinson's disease. Proc Natl Acad Sci U S A 105: 18578-18583.

88. Zhang S, Wang J, Song N, Xie J, Jiang H (2008) Up-regulation of divalent metal transporter 1 is involved in 1-methyl-4-phenylpyridinium $(\mathrm{MPP}(+))$-induced apoptosis in MES23.5 cells. Neurobiol Aging.
89. Liegeois S, Benedetto A, Garnier JM, Schwab Y, Labouesse M (2006) The V0ATPase mediates apical secretion of exosomes containing Hedgehog-related proteins in Caenorhabditis elegans. J Cell Biol 173: 949-961.

90. Nelson N, Harvey WR (1999) Vacuolar and plasma membrane protonadenosinetriphosphatases. Physiol Rev 79: 361-385.

91. Techau ME, Valdez-Taubas J, Popoff JF, Francis R, Seaman M, et al. (2007) Evolution of differences in transport function in Slc1la family members. J Biol Chem 282: 35646-35656.

92. Lin YT, Hoang H, Hsieh SI, Rangel N, Foster AL, et al. (2006) Manganous ion supplementation accelerates wild type development, enhances stress resistance, and rescues the life span of a short-lived Caenorhabditis elegans mutant. Free Radic Biol Med 40: 1185-1193. 